\title{
The applicability of dip moveout/azimuth moveout in the presence of caustics
}

\author{
Alison E. Malcolm¹, Maarten V. de Hoop², and Jérôme H. Le Rousseau ${ }^{3}$
}

\begin{abstract}
Reflection seismic data continuation is the computation of data at source and receiver locations that differ from those in the original data, using whatever data are available. We develop a general theory of data continuation in the presence of caustics and illustrate it with three examples: dip moveout (DMO), azimuth moveout (AMO), and offset continuation. This theory does not require knowledge of the reflector positions. We construct the output data set from the input through the composition of three operators: an imaging operator, a modeling operator, and a restriction operator. This results in a single operator that maps directly from the input data to the desired output data. We use the calculus of Fourier integral operators to develop this theory in the presence of caustics. For both DMO and AMO, we compute impulse responses in a constant-velocity model and in a more complicated model in which caustics arise. This analysis reveals errors that can be introduced by assuming, for example, a model with a constant vertical velocity gradient when the true model is laterally heterogeneous. Data continuation uses as input a subset (common offset, common angle) of the available data, which may introduce artifacts in the continued data. One could suppress these artifacts by stacking over a neighborhood of input data (using a small range of offsets or angles, for example). We test data continuation on synthetic data from a model known to generate imaging artifacts. We show that stacking over input scattering angles suppresses artifacts in the continued data.
\end{abstract}

\section{INTRODUCTION}

Data collected in the field are often not ideal for processing. For example, zero-offset data are important in seismic data processing, but limitations preclude collecting such data in the field. In general, we refer to methods to remedy this problem as data continuation or data mapping. Stolt (2002) gives an excellent description of why data continuation is necessary as well as a theory for performing data mapping with a constantbackground-velocity model. Patch (2002) gives an example of data continuation in medical imaging.

We introduce a theoretical tool to analyze data continuation in the presence of caustics, focusing on the particular examples of dip moveout (DMO) and azimuth moveout (AMO). The mathematical formulation of this tool is given in de Hoop et al. (2003b) and de Hoop and Uhlmann (personal communication, 2004); the purpose of our paper is to discuss its interpretation, application, and computation. In comparison with previous work, we pay special attention to the case in which caustics are present in the wavefield. In this case, the operator becomes, locally, significantly more complicated. The region where the complication occurs depends on the lateral heterogeneity of the velocity model used. Unlike traditional DMO and AMO operators, our operator changes along a profile; thus, the computation becomes significantly more complex in regions with complicated velocity structure. The practical value of this tool comes in constructing the near-offset data in regions where the velocity model is complicated.

We use the term data continuation to describe any act of computing data that have not been collected in the field. Early examples of data continuation using a partial differential equation can be found in Goldin (1994) and Goldin and Fomel (1995). Azimuth moveout is a special case of data

\footnotetext{
Manuscript received by the Editor February 17, 2003; revised manuscript received July 9, 2004; published online January 14, 2005.

${ }^{1}$ Colorado School of Mines, Center for Wave Phenomena, Department of Geophysics, 1500 Illinois Street, Golden, Colorado 80401. E-mail: amalcolm@dix.mines.edu.

${ }^{2}$ Colorado School of Mines, Center for Wave Phenomena, Department of Mathematics and Computer Science, 1500 Illinois Street, Golden, Colorado 80401. E-mail: mdehoop@dix.mines.edu.

${ }^{3}$ Formerly Colorado School of Mines, Center for Wave Phenomena, Golden, Colorado: presently Université de Provence, Laboratoire d'Analyse, Topologie et Probabilités-CNRS UMR6632, Centre de Mathématiques et Informatique, 39 rue F. Joliot-Curie 13453, Marseille cedex 13, France. E-mail: jlerous@cmi.univ-mrs.fr.

(C) 2005 Society of Exploration Geophysicists. All rights reserved.
} 
continuation in which the desired output data are common acquisition azimuth data. It is described for a constant-velocity model (Biondi et al., 1998) as a transformation of data acquired at a particular offset and acquisition azimuth into (an approximation of) the data that would have been recorded at another offset and acquisition azimuth. Similarly, DMO is a special case of AMO, in which the output offset is simply set to zero, giving normal-incidence, zero-offset data as the output. Dip moveout theory has been developed in constant-velocity media and vertically varying velocity models [see Hale (1991) for an overview]. We show examples of DMO and more general continuation to illustrate our theory.

In our approach to data continuation, we compose a modeling and imaging/migration operator to construct an operator that computes the desired output data from the available input data. [In a constant-velocity model, our continuation operator can be thought of as a solution to the partial differential equation used by Goldin (1994) and Goldin and Fomel (1995).] This approach has been developed in the absence of caustics (e.g., constant-velocity and constant-velocity-gradient media) by Bleistein and Jaramillo (2000), Canning and Gardner (1996), and Stolt (2002). Other related approaches can be found in Hubral et al. (1996), Santos et al. (1997), and Tygel et al. (1998). Our theory extends these results by allowing for both lateral variations in the velocity model and caustics in the rayfield. The occurrence of caustics is not uncommon in heterogeneous media (White et al., 1988). Continuation plays the role of forward interpolation in the process of data regularization, as defined by Fomel $(1995,2003)$ and Stolt (2002), required for imaging or inversion.

To unfold the caustics, implicitly keeping track of the contributions from different branches of the caustic, we use the angle parameterization. In this parameterization the scattering angle, which is the angle between the source and receiver rays, takes the place of offset in the traditional parameterization and the dip angle takes the place of the midpoint. In the absence of caustics, a single mapping exists between the traditional parameterization and the angle parameterization. In the presence of caustics, a family of maps is required because the caustics do not unfold in the single map traditionally used. This is explained further by de Hoop and Brandsberg-Dahl (2000).

Data continuation is not a stand-alone process; rather, it exists within a larger framework. Imaging or inversion, migration velocity analysis, and offset continuation are closely dependent on one another; the operators that connect the three processes are the annihilators of the seismic data (Stolk and de Hoop 2002; Brandsberg-Dahl et al., 2003b).

We first outline how the continuation operators are constructed and then show three examples: DMO, AMO, and offset continuation in shot records. To illustrate the properties of the first two operators, we compute their impulse responses in both a constant-velocity model and a gas lens model. For the offset continuation section, we demonstrate the applicability of our theory by filling in missing offsets in synthetic shot records. We include three appendices with derivations of certain parts of this theory in constant-velocity media. Appendix A contains a derivation of the DMO impulse response in closed form. In Appendix B we derive an expression for the amplitudes of the data continuation operator. This result could also be applied in varying velocity models by using the rms velocity as a local approximation to the true velocity. In Appendix $\mathrm{C}$ we show that if the input and output data configurations are the same, the operator does not change the positions of reflections in the data.

\section{IMAGING, MODELING, AND RESTRICTION}

Data continuation is developed from the composition of three processes: imaging/migration, modeling, and restriction. By composition we mean applying one operator after another and simplifying the result, by integrating over intermediate phase variables, into a single operator. The continuation process is a single operation that computes data for the desired acquisition geometry from the original data. This is similar to the derivation of AMO (Biondi et al., 1998), in which the observed data are first migrated to form an image of the subsurface (imaging), from which data with another acquisition geometry are modeled (modeling). The output data are assumed to have a certain acquisition azimuth, which is accomplished with the use of a restriction operator.

Composing the operators, given the acquisition surface, requires that we impose certain conditions on the velocity model (e.g., removing grazing caustics, caustics that are both at and parallel to the acquisition surface). In our scheme, the first operator, the imaging operator, is a generalized Radon transform (GRT) [Miller et al. (1987); de Hoop and BrandsbergDahl (2000)]. The second operator models the data for all possible (continuous) source and receiver positions at the surface, using an image of the subsurface as input. The final operator restricts this modeling operator so that only the desired output data are modeled, rather than the complete data set. Composing the three operators yields a single operator that computes the desired output data directly from the input data. This composition is done in Appendix B for a general continuation operator in a constant-velocity (or mildly depth varying) model. All three operators are Fourier integral operators. (Fourier integral operators, which generalize Fourier integrals, can be used to solve wave equations. These operators are characterized by an amplitude and a phase, but the phase is not necessarily linear in the space variables as it is in Fourier transforms.) Enforcing so-called transversal composition of these operators results in a Fourier integral operator for the composite continuation operator (de Hoop et al., $2003 b)$. Such transversal composition may be obtained by imposing conditions (known as the Bolker conditions) on the velocity model, which exclude phenomena such as horizontal wave guiding and encompass the traveltime injectivity condition of ten Kroode et al. (1998). Subject to the Bolker conditions, we can carry out continuation of the reflections without introducing false reflections. The theory of Fourier integral operators (Treves 1980a, b; Hörmander 1985a, b; Duistermaat, 1996) involves symplectic geometry (the geometry of phase space), which is exploited to give the proper geometrical tools to assess these conditions.

Our framework is a generalization of the framework of DMO, such as in Hale (1991). In fact, in a homogeneous medium the Fourier integral operators of this analysis reduce to the Fourier transforms used by Hale to derive DMO. If, however, the velocity model deviates from the constant or constant-gradient cases, several changes in the theory become necessary. In such media, angle parameterization, as opposed 
to offset parameterization, is preferred, since it allows the unraveling of caustics. Angle parameterization, discussed in de Hoop et al. (1999), uses the scattering point, scattering angle, and (in three dimensions) scattering azimuth. Thus, for DMO we replace acquisition offset and acquisition azimuth by scattering angle and scattering azimuth. In two dimensions offset is replaced by scattering angle. This is done because the scattering angle can be used to uniquely specify a pair of rays, given the subsurface point and migration dip (direction normal to the reflector), whereas offset along with the same subsurface parameters does not uniquely determine a pair of rays. Likewise, we redefine AMO as a transformation from one acquisition azimuth and subsurface scattering angle to a new acquisition azimuth and new subsurface scattering angle.

To relate this parameterization to the acquisition coordinates, we split the traveltime function into branches, each branch being single valued, where all branches taken together give the complete set of traveltimes. The suppression of artifacts, or false reflections, in the presence of caustics requires the integration (or stacking) over neighborhoods of the scattering angle and scattering azimuth in the input data. By using a neighborhood, we are able to discriminate between true features and artifacts through the use of multiple samplings of the same subsurface point. Although the data will sample the same subsurface point more than once, the data are not simply redundant; multiple samples, with different scattering angles, are required to suppress artifacts in the final image. In classical DMO, offset, which is used for parameterization, is held constant. In complex media, such a parameterization is not only inadequate but it also does not allow a straightforward discrimination between events and artifacts. For examples of procedures to suppress artifacts in imaging, see BrandsbergDahl et al. (2003a). Artifacts are classified in Stolk (2001).

To reduce the amount of numerical computation required, as many phase variables as possible should be integrated out (this is done for a constant-velocity medium in Appendix B). In general, at least one phase variable (frequency) will always remain in the final operator. In some cases-for example, 3D DMO in constant media (Bleistein et al., 2000, p. 326)additional phase variables must remain in the final operator, as explained in Appendix A of de Hoop et al. (2003b).

\section{DMO}

For DMO, finite-offset data are used in the imaging step, after which zero-offset (normal-incidence) data are modeled. When the zero-offset modeling is done by restricting a multiple-offset modeling operator to zero offset, data from nonnormal incidence are also modeled. We construct an exploding-reflector modeling operator (Loewenthal et al., 1976; Claerbout, 1985) and use it in place of the restricted modeling operator. The exploding-reflector modeling operator models only a single ray from the scattering point to the surface point. (From this point onward we refer to this single ray as the zero-offset ray and the associated data as zero-offset data.) This means that for DMO the composition of three operators (imaging, modeling, and restriction) is reduced to a composition of only two (imaging and exploding-reflector modeling).
Using the exploding-reflector modeling operator rather than the full zero-offset modeling operator results in a different amplitude for the final transformation to zero offset (TZO). A partial explanation for this is that the explodingreflector operator models the data along a single ray as if the wave speed of the medium were half its true value, while the zero-offset modeling operator models two rays (up- and downgoing) in the true medium with coincident surface and subsurface positions. The amplitude of the exploding-reflector modeling operator differs from that of the restricted multipleoffset modeling operator (RMO); the RMO models a ray that travels from the surface to the subsurface applying geometrical spreading, followed by modeling a ray from the subsurface to the surface applying geometrical spreading once again (resulting in the geometrical spreading squared). In contrast, the exploding-reflector modeling operator considers the geometrical spreading for just one ray with half the wave speed along the path.

\section{Homogeneous model}

For any operator, we can construct a table relating the input parameters to the output parameters. In migration, for example, this table would relate a point in the subsurface (the output of migration) to the source position, receiver position, and two-way traveltime (the input to migration). The table can be parameterized in different ways. For example, midpoint and offset, or source position and scattering angle, can be used in place of the source and receiver positions. Fixing different sets of these parameters allows us to plot cross-sections of this table. (We use the term cross-section as analogous with the cross-sections of a function. The cross-sections of a twovariable function, $f(x, y)$ say, are the graph $\left(y, f\left(x_{0}, y\right)\right)$ with $x_{0}$ a fixed value and the graph $\left(x, f\left(x, y_{0}\right)\right)$ with $y_{0}$ a fixed value.) As a first example, we plot the standard migration ellipse in Figure 1; in this case the midpoint, offset, and traveltime are fixed, giving us a particular cross-section of the table. Similarly, we can fix the source point $s$, scattering angle $\theta$, and traveltime $T$ giving what we call an angle isochron. (A full list of symbols is given in Table 1.) An example of an

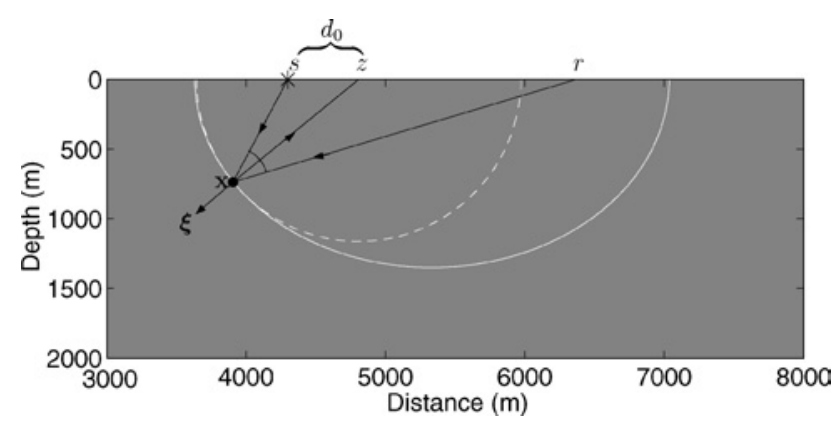

Figure 1. Offset DMO in a constant-velocity medium $(v=$ $1.7 \mathrm{~km} / \mathrm{s}$ ). The solid curve is a finite-offset isochron (fixed midpoint, offset, and traveltime $\left.t_{r}+t_{s}\right)$; the dashed curve is an exploding-reflector isochron (fixed zero-offset surface point $z$ and time $t_{0}$ ); the black lines are the rays. The black dot shows the location of the scattering point, which is the same as that marked by a dot in Figure 2. DMO maps from the finite-offset isochron to the exploding-reflector isochron, keeping the scattering point and the direction (migration dip) $\boldsymbol{\xi}$ fixed. 
Table 1. Table of symbols. In general, quantities with a subor superscript $s$ refer to source quantities; $r$ refers to receiver quantities. Bold symbols are vectors, and an underlined symbol is a function of other variables.

Symbol Meaning

\begin{tabular}{ll}
\hline$A(\mathbf{s}, \mathbf{x}, \mathbf{r})$ & Amplitude of the Green's function \\
$\mathbf{B}_{k l}$ & Second fundamental tensor \\
$c$ & Smooth background velocity \\
$d_{0}$ & Distance from source to zero-offset surface \\
$E_{v}, E_{\theta}, E_{\psi}$ & position \\
$F$ & Set of $\nu, \theta, \psi$ values \\
$F^{*}$ & Modeling operator \\
$G(\mathbf{r}, t, \mathbf{x})$ & Imaging/migration operator \\
& Green's function for singularities from $\mathbf{x}$ to $\mathbf{r}$ in \\
$\mathbf{H}$ & time $t$ \\
\multicolumn{1}{c}{ Hessian of second derivatives for stationary } \\
$h, \mathbf{h}$ & phase \\
$I$ & Half-offset \\
$\mathbf{J}$ & Left inverse of modeling operator \\
& Jacobian of the variable transformation \\
$k_{1}$ & $x_{1}, x_{2} \rightarrow \eta_{1}^{\prime}, \eta_{2}^{\prime}$ \\
\hline & Symbol to simplify calculations in
\end{tabular}

Symbol to simplify calculations in Appendix C

$N \quad$ Normal operator $F^{*} F$

$O_{r} \times O_{s} \quad$ Set of source and receiver positions

$P(\mathbf{x}, \mathbf{y}) \quad$ Parametrix (approximate inverse) of normal operator

p (q) Vectors from scattering point to source (receiver)

$S_{s}^{2} \quad$ Sphere of source ray directions

$S \quad$ Source position

$T \quad$ Two-way time

$T(\mathbf{x}, \mathbf{y}) \quad$ Traveltime function for ray traveling from $\mathbf{x}$ to $\mathbf{y}$

$t_{s} \quad$ Time along source ray

$t_{0} \quad$ One-way zero-offset time

$\mathbf{v} \quad v$ in input variables rather than output variables

$X \quad$ Set of subsurface scattering points

$\mathbf{x} \quad$ Subsurface position

$\mathbf{x}_{0} \quad$ Stationary point for $x$-integration

y Midpoint

$z, \mathbf{z} \quad$ Surface position of zero-offset ray

$\boldsymbol{\alpha}^{s} \quad$ Source ray direction

$\Gamma$

$\delta c$

$\boldsymbol{\eta}^{\prime}$

$\theta_{s}$

$\theta_{0}$

$\Lambda$

$\mu_{L S}$

$\nu$

$\xi$

$\sigma_{1}^{\prime}, \sigma_{2}^{\prime}$

$\varphi_{\alpha}$

$\varphi_{\alpha}$

$\psi_{\alpha}$

$\psi$

$\omega^{0}$

Spatial gradient of traveltime function

Velocity contrast

Fourier variable associated to midpoint

Scattering angle

Angle between surface and source ray

Angle from zero-offset ray to surface

Full amplitude of normal operator

Amplitude factor for normal operator

Normalized $\Gamma$ (migration dip)

Migration dip

Coordinates on level set of $T$

Parameter to fix which level set of $T$ we are on

Phase function

Spherical angle measured clockwise from $x$

Scattering azimuth

Spherical angle measured downward from $x-y$-plane

Azimuthal vector

Angular frequency

Stationary point in $\omega$ angle isochron is shown in Figure 2. In this construction, the receiver position and thus the midpoint and offset are variable. By fixing $s$ at the surface and $\theta$ at depth, we look at a different level set of the table; not surprisingly, an angle isochron has a different shape than a traditional isochron. Neither of these isochrons illustrates the DMO operator; rather, they show the shape of the migration or imaging operator for the particular velocity model. The dependence of the angle isochron on angle is shown in Figure 7 of de Hoop et al. (2003b).

Fomel and Prucha (1999) compute the Cheops pyramid in both the offset and angle domains. This is equivalent to fixing $\mathbf{x}$, the subsurface point, in the migration table and then plotting midpoint, offset, and traveltime. This pyramid and the common-angle diffraction curves are shown in Figure 3. The data continuation involves filling in the missing portions of the pyramid using the parts that remain. See Fowler (1998) and Sava and Fomel (2003) for more details on the pyramids.

Traditionally, the impulse response of the DMO operator is computed for any midpoint by using common-offset data containing a single Dirac-like impulse as input to the DMO algorithm. This is equivalent to computing-from fixed offset,

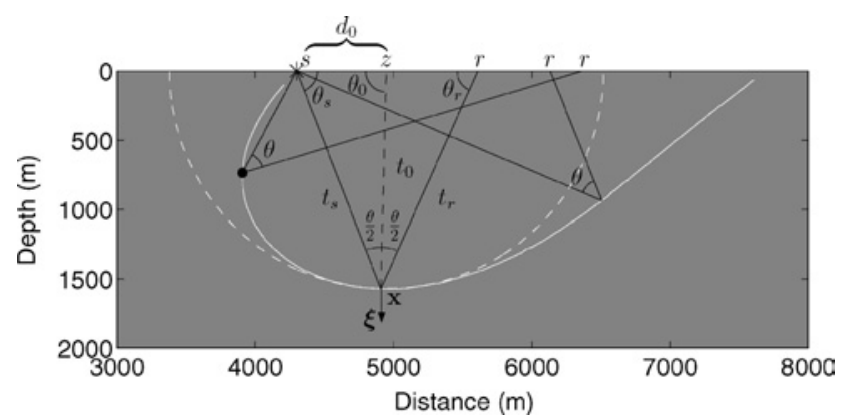

Figure 2. Angle DMO in a constant-velocity medium $(v=$ $1.7 \mathrm{~km} / \mathrm{s}$ ). The black lines are rays; the dashed white line is the exploding-reflector isochron; the solid white line is the angle isochron. The black dot is the scattering point from Figure 1 ; this point will have a different contribution to the operator illustrated in this figure than it does to the operator illustrated in Figure 1. The angle $\theta_{0}$ between the zero-offset ray and the surface is variable; it depends on the particular value of the scattering angle. This illustration shows $\theta_{0}$ close to $90^{\circ}$, which generally is not the case. The notation shown in this figure is used throughout the text and in Appendix A, where the impulse response is computed.
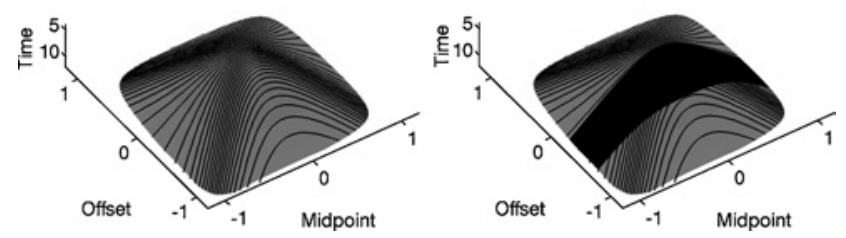

Figure 3. Cheops pyramid. The lines represent curves of constant scattering angle. The blacked-out region illustrates the removal of some offsets, which will be filled in by the continuation procedure. This plot shows several data surfaces with fixed depth point $\mathbf{x}$ and scattering angle. 
source position (or equivalently midpoint), and traveltimethe zero-offset traveltime as a function of the distance from the source position to the zero-offset source-receiver position ( $d_{0}$ in Figure 1$)$. Figure 1 shows the traditional DMO construction. For the initial source location, offset, and traveltime, we compute an isochron (solid white line). From this isochron, the scattering point $\mathbf{x}$ and migration dip $\xi$ are used to shoot a zero-offset ray. This ray is normal to the exploding-reflector isochron (dashed line), which is a circle centered at the point $z$. Thus, DMO maps data points from a finite-offset isochron to the exploding-reflector isochron matching the scattering point and migration dip.

To compute the impulse response in the angle domain (using the notation of Figure 2), we fix $\theta, s$, and the traveltime $T=t_{s}+t_{r}$ and compute the one-way zero-offset traveltime $t_{0}$ as a function of the distance $d_{0}$ from the source position $s$ to the zero-offset source-receiver position $z$. In a constantvelocity medium, this impulse response can be computed in closed form (see Appendix A). Figure 4 shows the impulse response for angle DMO, as derived in Appendix A. This impulse response is the zero-offset traveltime as a function of the distance from the source position to the zero-offset position (the distance $d_{0}$ in Figure 2). The solid line is the closed-form solution, and the points are computed numerically with ray tracing; the spacing between the points is constant (increment $5.73^{\circ}$ ) in the take-off angle $\theta_{s}$ (defined in Figure 2).

\section{Gas lens model}

The gas lens model consists of a vertical velocity gradient $\left(0.45 \mathrm{~s}^{-1}\right)$ beginning at $1600 \mathrm{~m} / \mathrm{s}$ with a low-velocity circular lens with Gaussian parameter variations (maximum velocity contrast $800 \mathrm{~m} / \mathrm{s}$ ) located at lateral position $4600 \mathrm{~m}$ and depth $600 \mathrm{~m}$ with a diameter (Gaussian standard deviation) of $600 \mathrm{~m}$. This model, introduced by Brandsberg-Dahl et al. (2003a), is based on a feature in the BP Valhall field. Throughout this subsection, we compare results for this model with those of a constant-velocity gradient model (the same as the lens model but without the lens). The background shading of Figures 5 and 6 depicts the velocity model, with darker shading indicating higher velocity.

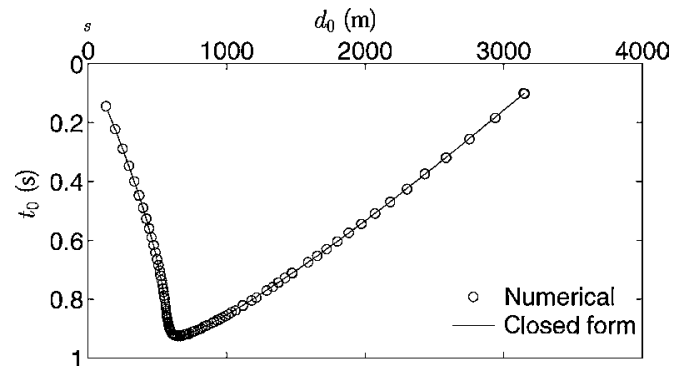

Figure 4. DMO impulse response for a constant-velocity medium, a scattering angle of $45^{\circ}$, a velocity of $1.7 \mathrm{~km} / \mathrm{s}$, and $T=2 \mathrm{~s}$. The spacing of the points is constant in take-off angle at the source ray (increments of $5.73^{\circ}$ ). Areas of the impulse response with denser sampling, which are regions of high curvature, can be expected to have relatively high associated amplitude. The lowercase $s$ is the position of the source.
In Figure 5, we show the relationship between the shape of the angle isochron in the lens model (solid line) and that in the constant-gradient model (dashed line). The introduction of the lens adds complicated structures to the isochron not present in the constant-gradient response, but the gross shape of the main branch remains similar. Figure 6 illustrates the origin of these features by showing the fixed-scatteringangle rays that build various portions of the isochron. Even this simple velocity variation introduces large complications in these angle isochrons, caused by the complicated geometry of the rayfield in the vicinity of the lens.

If traditional DMO were performed on data from this model using the incorrect assumption of a constant velocity gradient, the results could contain significant errors. One source of these errors is that the computed zero-offset time would be calculated for zero-offset rays originating on the dashed curve rather than the more complicated solid one in Figure 5. It is possible to create an operator that corrects such an error. By composing inverse DMO in the constantgradient medium with DMO in the more complicated lens model, an operator can be constructed to reapply DMO with a more accurate velocity model (e.g., for velocity analysis). The idea of residual DMO has been developed by Alkhalifah and de Hoop (personal communication, 2004) in homogeneous anisotropic media. The composed operator is more efficient to apply than inverse DMO in the wrong model followed by DMO in the correct model.

To suppress artifacts inherent in the GRT in the presence of caustics (Brandsberg-Dahl et al., 2003a), it is necessary to stack over a range of scattering angles. To illustrate the cancellation of these artifacts, we plot the impulse response in the lens model for three different scattering angles $\left(40^{\circ}, 45^{\circ}\right.$, and $50^{\circ}$ ) in Figure 7 . For these three scattering angles, the gross structure present in the constant-gradient case (dashed line) remains essentially unchanged at the three different angles, while the more complicated structures change more rapidly. Small changes in the scattering angle result in differences in the maximum $t_{0}$ and $d_{0}$ as well as detailed changes in the small loop structure located at $t_{0} \approx 1 \mathrm{~s}, d_{0} \approx-700 \mathrm{~m}$. Because of this, stacking over angle will result in a smaller contribution both at large times and in the loop structure than from regions in which the impulse response does not change.

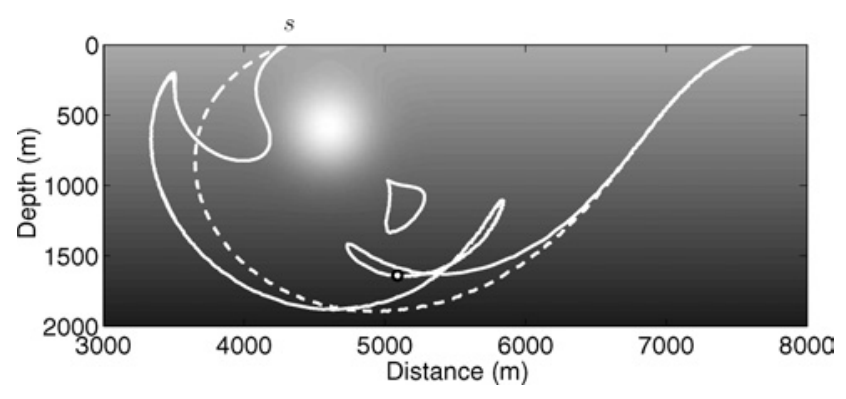

Figure 5. Angle isochron in the lens model; scattering angle is $45^{\circ}, T=2 \mathrm{~s}$, source is at $4300 \mathrm{~m}$. The solid line uses the lens model, and the dashed line uses the constant-gradient model without the lens. The background shading portrays the velocity function for the lens model, with darker shading denoting higher velocity. 
The composition of the imaging operator with the exploding-reflector modeling operator can be illustrated by again matching the migration dip and scattering point on a finite-offset isochron with those on a conventional explodingreflector isochron. The counterpart of Figure 1 in the presence of a lens is shown in Figure 8. For lateral heterogeneity, the shape of the isochron is evaluated for a particular midpointoffset pair and will change with midpoint, unlike its behavior for a laterally homogeneous medium.

In the lens model (Figure 8), the finite-offset isochron exhibits two regions of multipathing, indicated by the triplications in the isochron. The exploding-reflector isochron, which is a snapshot of the wavefront as it passes through the lens, exhibits only a single region of multipathing. The contribution to the DMO operator comes from the points where the two isochrons meet tangentially. The associated rays are the same as those that meet at the point marked with a circle on the angle isochron of Figure 5.

\section{DATA CONTINUATION AND AMO}

In this more general case, we compose the GRT as the imaging operator, with high-frequency, single-scattering modeling and a restriction operator specific to the application. This restriction operator determines the form of the output data. Although the theory applies more generally, the main ideas may be understood based on the calculations in a constant-velocity model given in Appendix B, which also includes a computation of the amplitudes.

When attempting to fill in missing data, it is important that the computed data agree well with the true data. Therefore, the continuation operator must leave the data unchanged
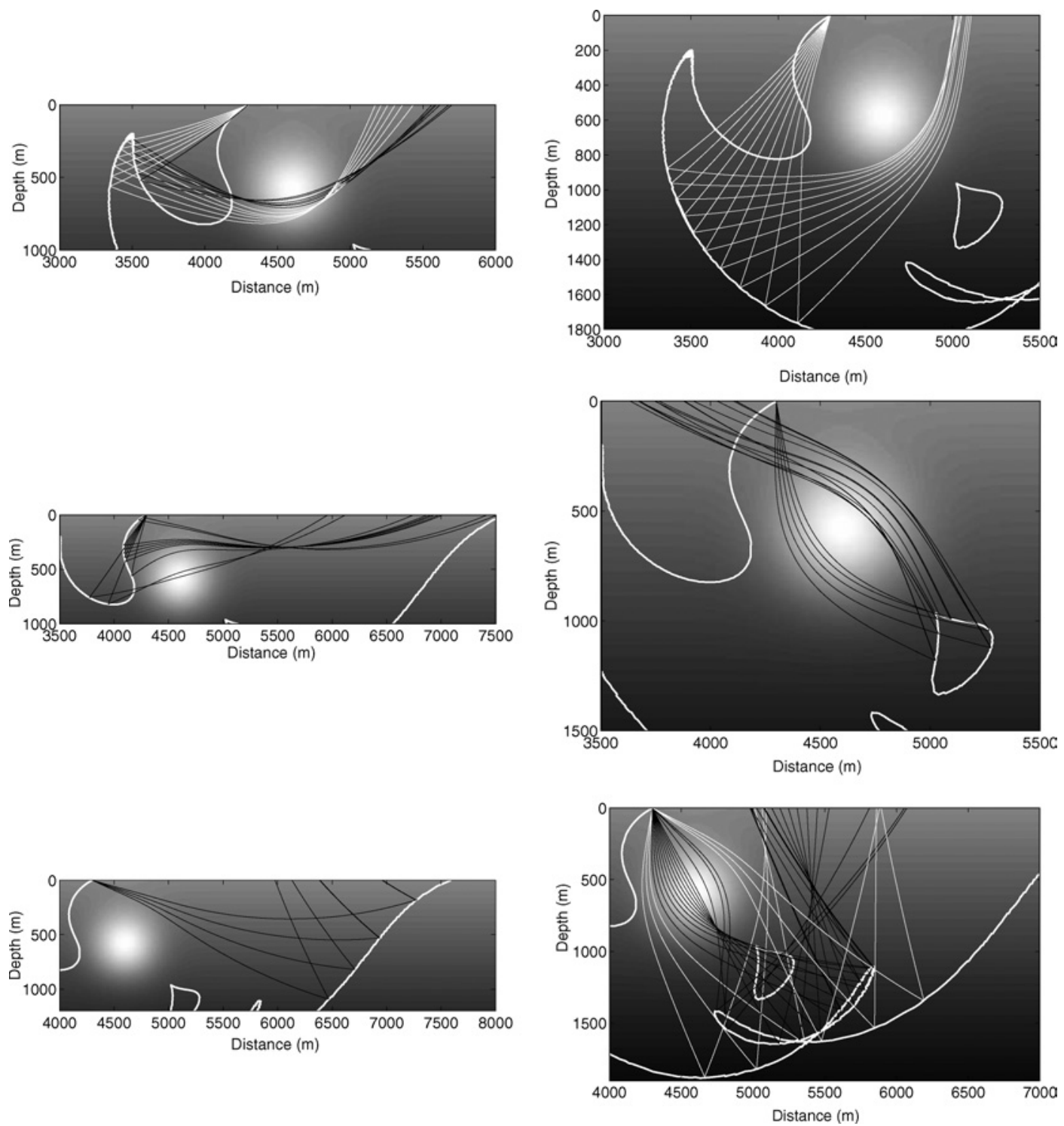

Figure 6. Raypaths in the lens model for a scattering angle fixed at $45^{\circ}$. This illustrates the origin of the complicated structure of the angle isochron by showing the rays used to compute it. Where both white and black rays are present, white rays come from the main branch, while black rays come from inner (closer to the lens) branches. Each panel shows a different region of the same angle isochron. 
if the input and output source-receiver positions are the same. We illustrate this is the case in Appendix C, by showing that the application of a modeling operator after an imaging operator (for the common-offset case) results in a so-called pseudodifferential operator, a partial definition of which is a forward and inverse Fourier transform with an additional multiplication in the Fourier domain.

Data continuation applies in both two and three dimensions. From the 3D case, a transition from three to 2.5 dimensions is possible, although the 2D case does not follow directly from the 3D theory. The methodology for the transformation to 2.5 dimensions in terms of Fourier integral operators is discussed in Foss et al. (2003).

\section{Homogeneous model}

Biondi et al. (1998) construct AMO as a mapping of data collected at a given offset and acquisition azimuth to data that would have been collected at a different offset and acquisition azimuth. We construct AMO as a mapping from an input scat-

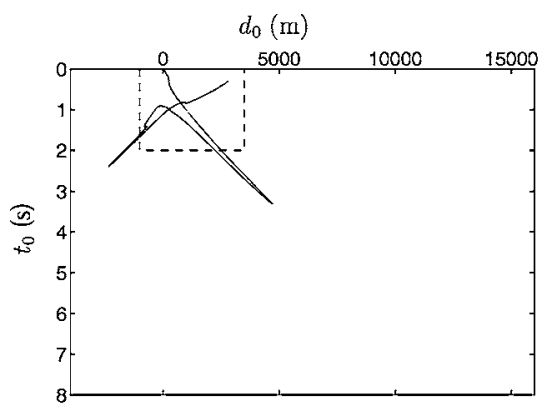

a)

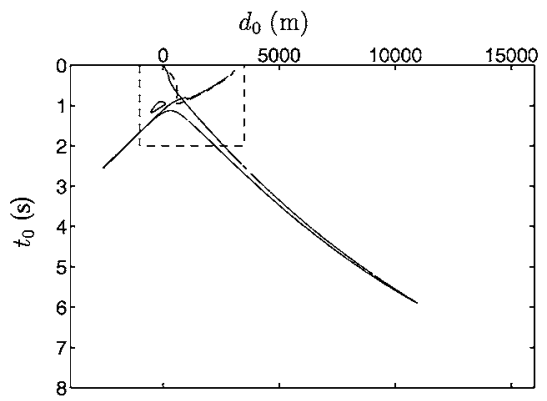

c)

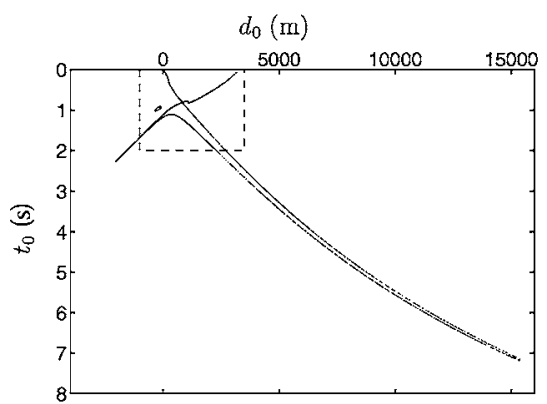

e)

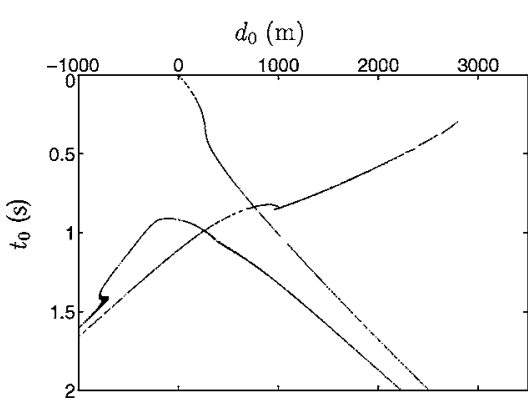

b)

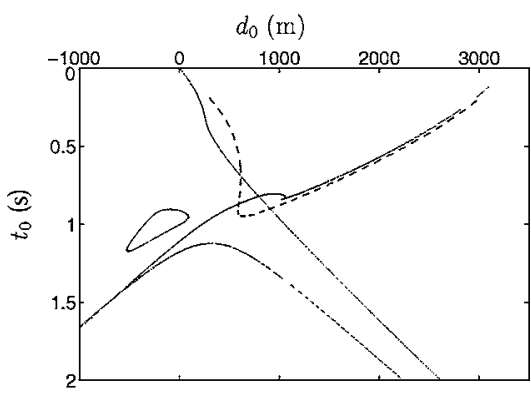

d)

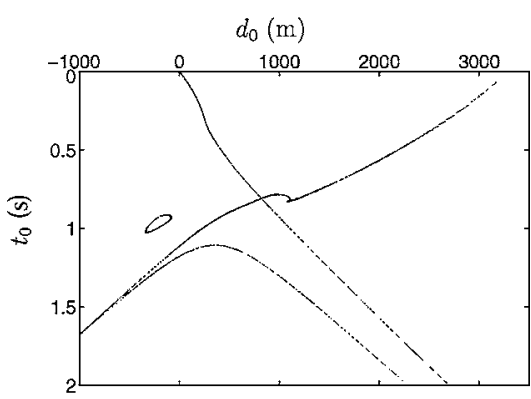

f)
Figure 7. Angle DMO impulse responses for the lens model for scattering angles of (a, b) $40^{\circ}$, (c, d) $45^{\circ}$, and (e, f) $50^{\circ}$. The dashed box in (a), (c), and (e) outlines the region shown in (b), (d), and (f). The dashed curves in (c) and (d) are the impulse responses computed in the constant vertical velocity gradient that makes up the background of the lens model. tering angle and scattering azimuth to an output surface line (acquisition azimuth) and scattering angle.

We compute the angle isochrons, as well as our impulse response, in three dimensions. In Biondi et al. (1998), the impulse response is the output traveltime as a function of the change in midpoint between the input and output data. For our method the impulse response is again the output traveltime but is now a function of the initial direction of the source ray, given in terms of the spherical angles $\psi_{\alpha}$ (angle clockwise from $x_{1}$ ) and $\varphi_{\alpha}$ (angle measured downward from the $x_{3}=0$ plane). In three dimensions, the angle isochron is an extension of the 2D angle isochron (Figure 2). The AMO operator takes the initial source and receiver rays and rotates the plane in which they are contained about the zero-offset ray to the desirection. The output rays are then computed in is new direction with a defined scattering angle, keeping the -offset ray fixed. A closed-form expression for this surface is derived in Appendix B of de Hoop et al. (2003b); it is shown in Figure 9 of that paper.

\section{Gas lens model}

To illustrate the complications that arise in a laterally heterogeneous model, we compute the isochron and AMO impulse response for a model containing a low-velocity lens, as done for the constant-velocity model. The model is similar to that used in the $2 \mathrm{D}$ case; it is scaled down in size to reduce computation cost. The model consists of a spherical lens with Gaussian parameter variations of $100 \mathrm{~m}$ diameter (standard deviation of Gaussian); center at $x_{1}=600 \mathrm{~m}, x_{2}=600 \mathrm{~m}, x_{3}=$ $100 \mathrm{~m}$; and a maximum velocity contrast of $800 \mathrm{~m} / \mathrm{s}$. The model extends to $x_{1}=2400 \mathrm{~m}$, $x_{2}=2400 \mathrm{~m}, x_{3}=800 \mathrm{~m}$ with an initial velocity of $1600 \mathrm{~m} / \mathrm{s}$ and a vertical gradient of $0.45 \mathrm{~s}^{-1}$.

The angle isochron shown in Figure 9 exhibits a gross shape similar to that in the constant-velocity case. Just as with the 2D angle isochron (Figure 5), the 3D version has a complicated shape in which we recognize the presence of caustics. Figure 10 shows the AMO impulse response, which bears little resemblance to that for the constant-velocity model. The complications in the AMO impulse response and angle isochrons for the lens model are significantly greater for the 3D problem than for the $2 \mathrm{D}$ one.

\section{SYNTHETIC DATA EXAMPLE}

The following example of data continuation uses the same 2D lens model as for the DMO impulse response calculations, with the addition of a reflector from which synthetic reflection data are computed. The model is shown in Figure 11, and further details on its properties can be found in BrandsbergDahl et al. (2003a). 
The goal of this example is to reconstruct data that are missing from the original acquisition. To demonstrate this, we have removed all traces with offsets between 0 and $500 \mathrm{~m}$ from the synthetic reflection data set described above. This is illustrated in Figure 3, where we have deleted a set of offsets from the pyramid. The algorithm works in the angle domain but reconstructs missing offsets.

We then use equation B-27 for general continuation to fill in the data that have been removed. Although the theory is developed in the common-shot domain, the algorithm accesses the traces at random. The $2 \mathrm{D}$ algorithm is as follows:

\author{
for each subsurface point $\mathbf{x}$ \\ for each migration dip direction $\boldsymbol{\xi} /\|\boldsymbol{\xi}\|$ \\ for each input scattering angle $\theta_{i}$ \\ shoot scattering rays to surface \\ if the rays reach surface within the data range \\ get data sample at this position \\ for each output scattering angle $\theta_{o}$ \\ shoot scattering rays to surface \\ if these rays are in missing data range \\ add contribution to this point in missing data
}

Although this algorithm works, it is far from the most efficient one possible. For example, we shoot many of the rays more than once, repeating for the output data computations already done for the input data. An example of a more efficient way to do the computations is based on the escape equations developed by Sethian (2002).

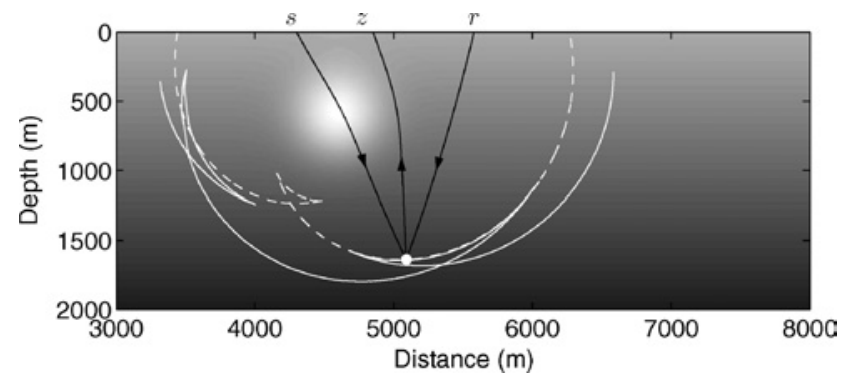

Figure 8. Fixed-offset isochrons in the lens model. The solid white line is the finite-offset isochron, and the dashed line is the exploding-reflector isochron; $s, r$, and $z$ are the source position, receiver position, and surface point for the zero-offset ray, respectively. Compare this figure with Figure 1, which shows the isochrons in the constant-velocity medium.

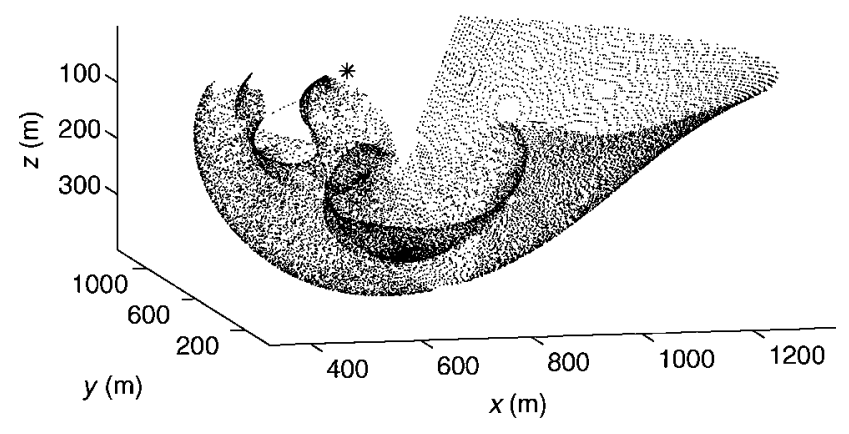

Figure 9. Angle isochron in the lens model for a source position indicated by the asterisk. This is an extension to three dimensions of the angle isochron shown in Figure 5.
Figure 12 shows the results of this procedure applied to the data in three synthetic shot records for the model in Figure 11. The top panels show a shot record reconstructed away from the influence of the lens. At this position the event is filled in correctly. Because the event is so simple at this point, interpolation would work just as well. The second row of panels down shows the reconstruction of a shot record on the edge of the lens. Traces with events from both the dipping and flat portions of the reflector are missing, making the reconstruction more difficult. The reconstruction is reasonably successful, although there are some amplitude errors. The third row of panels shows a shot record for the shot right over the lens. We have removed the traces containing the caustic and are attempting to fill them in with the algorithm. Again, the reconstruction is not perfect, but it is considerably better than what would have been achieved using conventional interpolation schemes. The data shown have been bandpass filtered to match the frequency content of the original data. We expect some amplitude inaccuracies because the amplitudes are obtained from equation B-27, which are for a constant-velocity model, whereas this model is clearly more complicated. In these examples we have corrected for only the obliquity factor $\left(\left|\cos \theta_{i}\right|^{2}\right)$.

The single shot record at the bottom shows the same computation as above it with a range of only $2^{\circ}$ in scattering angle for the input data (compared with the section above it, which uses all the available data). A particularly high-amplitude artifact appears just below the latest true event in this section.

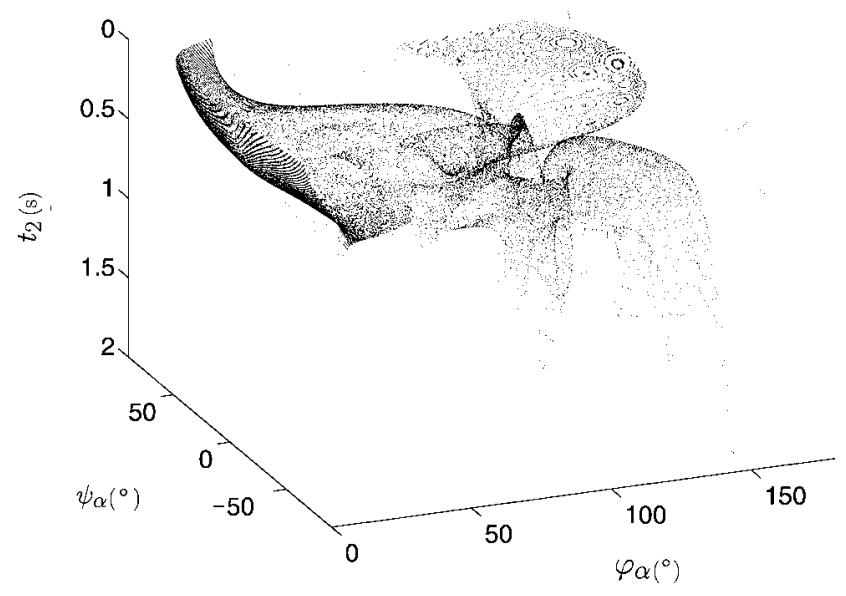

Figure 10. The AMO impulse response for the lens model. Compared with the impulse response for DMO (2D case) in Figure 7, the 3D nature of AMO introduces further complications in this impulse response.

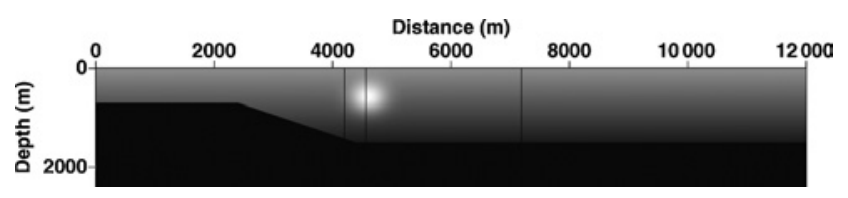

Figure 11 . Velocity model for a synthetic data set. This model is the same as that used for the 2D DMO examples with the addition of the reflector. The vertical lines indicate the location of the shot points for which shot records are shown in Figure 12. 

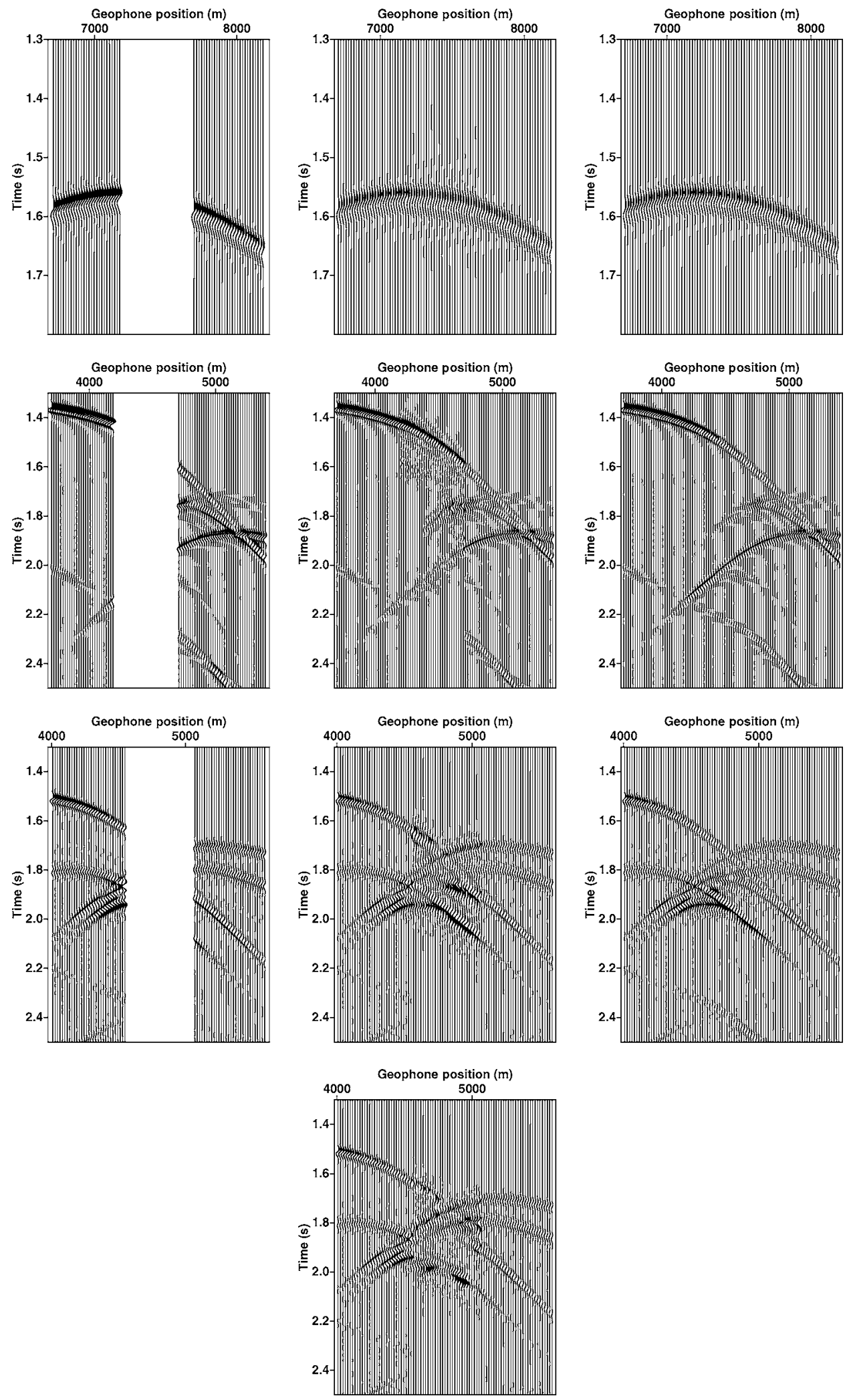

Figure 12. In each row, a different shot record has a block of traces removed and then reconstructed. The left column shows the input data, the middle column is the reconstructed data, and the right column is the actual full synthetic shot record. The extra section shown at the bottom illustrates the presence of artifacts when insufficient stacking is done over the input scattering angle. The position of the first shot record is at $s=7188 \mathrm{~m}$, the second is at $s=$ $4200 \mathrm{~m}$, and the third is at $s=4560 \mathrm{~m}$. These locations are denoted with vertical lines in the velocity model (Figure 11). 

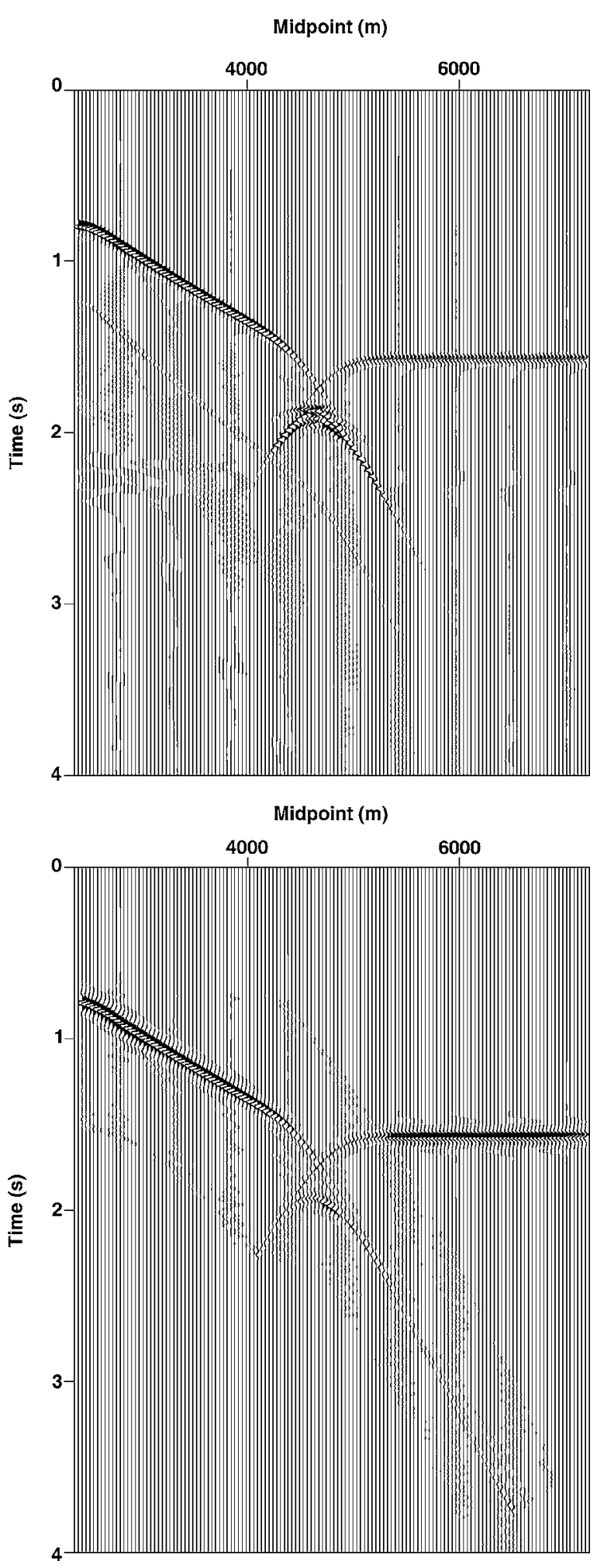

Figure 13. The top panel shows the smallest offset available in the synthetic data set $(6 \mathrm{~m}$ offset). The bottom panel is the exploding-reflector data computed from the portion of the synthetic data set with offsets between -100 and $100 \mathrm{~m}$ removed.
This example can be generated much more quickly than the other images because so few input angles are used. The other panels most likely use more angles than necessary, so the optimal data quality and computational time trade-off is probably somewhere in between the two.

Finally, to mimic the DMO discussion above, we show in Figure 13 the exploding-reflector data computed from the original data set (with offsets from -6 to $7 \mathrm{~km}$ ) while removing offsets from -100 to $100 \mathrm{~m}$. The smallest offset data (offset of $6 \mathrm{~m}$ ) from the true data set are shown for comparison. This illustrates the difference between zero-offset modeling and exploding-reflector modeling.

\section{CONCLUSIONS}

We have described and illustrated a method for sourcereceiver continuation of seismic data in the presence of caustics. In the absence of caustics, the method reduces to a form of offset continuation. The computational complexity of this method depends on the geologic complexity and varies in space as the geology changes. In the most complex situation a table must be constructed relating the subsurface and surface parameters, and a search must be performed in this table; the computational complexity depends on the algorithms used to perform these steps. Both DMO and AMO are examples of data continuation that can be obtained from the continuation framework presented here. It is possible to continue data only when the subsurface point and reflector orientation generating the data point are sampled in the original data set. Thus, the issue of illumination is directly related to data continuation. Similarly, data continuation provides a framework for estimating the smallest necessary data set by using a combination of acquisition and data continuation to construct data needed for migration. An example of when a general data continuation theory is important is in reducing a multiazimuth data set with AMO to a common-azimuth data set for efficient migration through downward continuation.

\section{ACKNOWLEDGMENTS}

We would like to thank Henri Calandra for his help in writing the first version of the continuation code. We are also grateful to Stig-Kyrre Foss and Sverre Brandsberg-Dahl who provided some of the subroutines in the continuation code used to generate the examples shown. We would also like

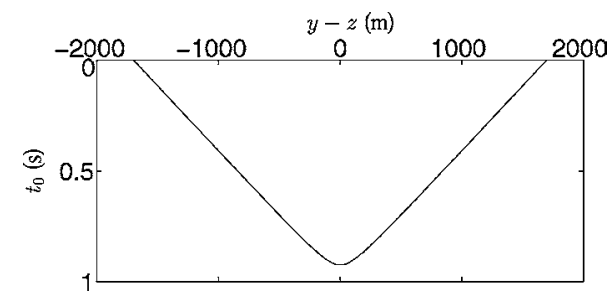

Figure 14. The DMO impulse response showing the zerooffset traveltime as a function of the distance from the midpoint to the surface position of the zero-offset ray. Crossplotting these quantities makes the impulse response symmetric in a constant-velocity model. This symmetry disappears if the velocity model is not symmetric. 
to thank Ken Larner and John W. Stockwell for their assistance in the preparation of this manuscript as well as Sergey Fomel and the four anonymous reviewers who all gave excellent suggestions for enhancing the relevance of the results presented in this paper. In particular, the equations in Appendix A for the symmetric impulse response were suggested by Sergey Fomel. This work was supported by the sponsors of the Consortium Project on Seismic Inverse Methods for Complex Structures at the Center for Wave Phenomena, by Civilian Research and Development Foundation grant RG1-2362-NO-02, and by Total.

\section{APPENDIX A}

\section{IMPULSE RESPONSES}

\section{DMO}

For a constant-velocity model one can derive, in closed form, the shape of the angle-domain DMO impulse response. To do this, we fix the source position $s$, the scattering angle $\theta$, and the two-way traveltime $T$ and compute the output time $t_{0}$ and the source to zero-offset distance $d_{0}$. We use the notation defined in Figure 2.

Since we assume the traveltime to be fixed and the medium to have a constant velocity (straight rays), we have the following three relations (see Figure 2):

$$
\begin{aligned}
& \theta_{r}=\pi-\theta-\theta_{s}, \\
& \theta_{0}=\pi-\frac{\theta}{2}-\theta_{s}, \\
& T=t_{s}+t_{r} .
\end{aligned}
$$

From the law of sines we derive the following relationships:

$$
\begin{aligned}
t_{0} & =\frac{T \sin \theta_{s} \sin \theta_{r}}{\left(\sin \theta_{s}+\sin \theta_{r}\right) \sin \theta_{0}}, \\
d_{0} & =\frac{v T \sin \theta_{r} \sin \left(\frac{\theta}{2}\right)}{\left(\sin \theta_{s}+\sin \theta_{r}\right) \sin \theta_{0}},
\end{aligned}
$$

which gives both the one-way zero-offset traveltime $t_{0}$ and the distance $d_{0}$ between the source and the zero-offset sourcereceiver as a function of the source angle $\theta_{s}$. Figure 4 shows this impulse response ( $t_{0}$ as a function of $d_{0}$ ). In these expressions, $\theta$ and $T$ are fixed, while the other angles vary.

Although equations A-4 and A-5 are given in terms of $\theta_{s}$, $\theta_{r}$, and $\theta_{0}$, it is possible to fully determine both $t_{0}$ and $d_{0}$ in terms of only two angles. Using equation A-1 to substitute for $\theta_{r}$ and A-2 for $\theta_{s}$, equation A-4 simplifies to

$$
t_{0}=\frac{\sin ^{2} \theta_{0}-\sin ^{2}\left(\frac{\theta}{2}\right)}{2 \sin ^{2} \theta_{0} \cos \left(\frac{\theta}{2}\right)} .
$$

Using equations 44-46 of Fomel (2003) along with equation A-6 allows us to arrive at the expression

$$
y-z=\frac{v T \cos \theta_{0} \sin ^{2}\left(\frac{\theta}{2}\right)}{2 \sin ^{2} \theta_{0} \cos \left(\frac{\theta}{2}\right)},
$$

where $y=(s+r) / 2$ is the midpoint. The relation between our notation and Fomel's is $\gamma=\theta / 2, y-y_{0}=y-z, \alpha=\pi / 2-\theta_{0}$. Using equations A-6 and A-7 for the impulse response rather than equations A-4 and A-5 results in a symmetric impulse response, shown in Figure 14. The symmetry disappears in a nonsymmetric heterogeneous velocity model such as the lens model. Also, plotting $s-z$ rather than $y-z$ highlights the fact that we keep the source position $s$ fixed (i.e., we work in the common-shot domain).

Using equation A-7 along with the expression

$$
h=\frac{v T \sin \left(\frac{\theta}{2}\right)}{\sin \theta_{0}}
$$

for the half-offset $h$ allows us to follow Fomel (2003) to arrive at the DMO smile of Deregowski and Rocca (1981).

In a constant-velocity medium, one can also derive an expression for the AMO impulse response in closed form. This is done in Appendix B of de Hoop et al. (2003b), with the final result given in equation B.9.

\section{APPENDIX B ON AMPLITUDES}

\section{Modeling}

We begin with the kernel of the Born modeling operator [de Hoop et al. (2003b) their equation 7],

$$
\begin{aligned}
F[\delta c](\mathbf{r}, \mathbf{s}, t)= & \int_{X} \int_{0}^{t} G\left(\mathbf{r}, t-t^{\prime}, \mathbf{x}\right) 2 c^{-3}(\mathbf{x}) \partial_{t^{\prime}}^{2} \delta c(\mathbf{x}) \\
& \times G\left(\mathbf{x}, t^{\prime}, \mathbf{s}\right) \mathrm{d} t^{\prime} \mathrm{d} \mathbf{x} .
\end{aligned}
$$

In the above equation, $G\left(\mathbf{r}, t-t^{\prime}, \mathbf{x}\right)$ is the Green's function for the ray from the scattering point to the receiver and $2 c^{-3}(\mathbf{x})$ $\partial_{t^{\prime}}{ }^{2} \delta c(\mathbf{x}) G\left(\mathbf{x}, t^{\prime}, \mathbf{s}\right)$ is the contrast source at the scattering point, generated by the true source at the surface. The background velocity is denoted by $c(\mathbf{x}), \delta c(\mathbf{x})$ is the velocity perturbation that contains the locations of the reflectors, and $X$ is the set of scattering points in the subsurface. The notation $F[\delta c](\mathbf{r}, \mathbf{s}, t)$ indicates that the operator $F$ acts on the perturbation $\delta c$, with the result being dependent on the variables $(\mathbf{r}, \mathbf{s}, t)$. We use the full Born theory; the amplitudes will change if the Kirchhoff approximation is used in place of Born. For example, Biondi et al. (1998) use the Zhang-Black (Black et al. 1993) amplitudes, which are different from those used in this paper. The amplitude in equation 7.2.1 of Bleistein et al. (2000) should be compared with that of equation B-1. Equation 20 of Black et al. (1993) compares Black's DMO amplitudes and Hale's DMO amplitudes. Equation 7.6.36 of Bleistein et al. (2000) compares the Bleistein amplitudes with those of Hale (1991).

From this point onward we will assume that the background velocity $c$ of the medium is constant. Thus, the Green's function is given by

$$
G(\mathbf{y}, t, \mathbf{x})=\frac{1}{2 \pi} \int \frac{1}{4 \pi|\mathbf{y}-\mathbf{x}|} e^{-i \omega(t-T(\mathbf{x}, \mathbf{y}))} \mathrm{d} \omega,
$$

where $T(\mathbf{x}, \mathbf{y})=|\mathbf{x}-\mathbf{y}| / c$ is the traveltime between $\mathbf{x}$ and $\mathbf{y}$. The Green's function, as written in equation B-2, is the kernel of a Fourier integral operator (Stolk and de Hoop, 2002). To construct $F$, we substitute the two Green's functions into 
equation B-1, obtaining

$$
\begin{gathered}
F[\delta c](\mathbf{r}, \mathbf{s}, t)=\frac{1}{2 \pi} \int_{\mathbb{R}} \frac{1}{2 \pi} \int_{\mathbb{R}} \int_{0}^{t} \int_{X} \frac{2 c^{-3}\left(-\omega^{2}\right) \delta c(\mathbf{x})}{4 \pi|\mathbf{s}-\mathbf{x}| 4 \pi|\mathbf{x}-\mathbf{r}|} \\
\times e^{-i \omega\left(t-t^{\prime}-T(\mathbf{x}, \mathbf{r})\right)-i \omega^{\prime}\left(t^{\prime}-T(\mathbf{s}, \mathbf{x})\right)} \mathrm{d} \mathbf{x} \mathrm{d} t^{\prime} \mathrm{d} \omega \mathrm{d} \omega^{\prime} .
\end{gathered}
$$

Performing the integration with respect to $t^{\prime}$ results in $2 \pi \delta\left(\omega-\omega^{\prime}\right)$, so that the final operator is given by

$$
\begin{aligned}
& F[\delta c](\mathbf{r}, \mathbf{s}, t)=\frac{1}{2 \pi} \int_{\mathbb{R}} \int_{X} \frac{-\omega^{2} 2 c^{-3}}{16 \pi^{2}|\mathbf{s}-\mathbf{x}||\mathbf{x}-\mathbf{r}|} \\
& \quad \times e^{-i \omega(t-T(\mathbf{x}, \mathbf{r})-T(\mathbf{s}, \mathbf{x}))} \delta c(\mathbf{x}) \mathrm{d} \mathbf{x} \mathrm{d} \omega .
\end{aligned}
$$

\section{Imaging}

We use the adjoint operator (of the modeling operator) as the imaging-migration operator (first step toward inversion):

$$
\begin{aligned}
& F^{*}[u](\mathbf{x})=\frac{1}{2 \pi} \int_{\mathbb{R}} \int_{O_{r} \times O_{s}} \int_{\mathbb{R}_{+}} \frac{-\omega^{2} 2 c^{-3}}{16 \pi^{2}|\mathbf{s}-\mathbf{x}||\mathbf{x}-\mathbf{r}|} \\
& \quad \times e^{i \omega^{\prime}(t-T(\mathbf{x}, \mathbf{r})-T(\mathbf{s}, \mathbf{x}))} u(\mathbf{r}, \mathbf{s}, t) \mathrm{d} t \mathrm{~d} \mathbf{s} \mathrm{d} \mathbf{r} \mathrm{d} \omega^{\prime},
\end{aligned}
$$

where we use $\omega^{\prime}$ since we will shortly compose this operator with the modeling operator given above. We use $O_{r} \times O_{s}$ as the domain of source and receiver positions.

\section{Normal operator}

To obtain the correct amplitude characteristics of the output data, we construct the normal operator $N=F^{*} F$ so that we may use the left inverse, $\left(F^{*} F\right)^{-1} F^{*}$. To do this, we compose the modeling and imaging operators (recalling that $c$ is constant:

$$
\begin{aligned}
& N[\delta c](\mathbf{y})=\frac{1}{2 \pi} \int_{\mathbb{R}} \mathrm{d} \omega^{\prime} \frac{1}{2 \pi} \int_{\mathbb{R}} \mathrm{d} \omega \int_{O_{r} \times O_{s}} \int_{\mathbb{R}_{+}} \int_{X} \\
& \quad\left[\omega^{2} 4 c^{-6} A(\mathbf{s}, \mathbf{x}, \mathbf{r}) \omega^{\prime 2} A(\mathbf{s}, \mathbf{y}, \mathbf{r})\right. \\
& \left.\quad \times e^{-i \omega(t-T(\mathbf{r}, \mathbf{x}, \mathbf{s}))+i \omega^{\prime}(t-T(\mathbf{r}, \mathbf{y}, \mathbf{s}))}\right] \delta c(\mathbf{x}) \mathrm{d} \mathbf{x} \mathrm{d} t \mathrm{~d} \mathbf{s} \mathrm{d} \mathbf{r}
\end{aligned}
$$

where $A(\mathbf{s}, \mathbf{x}, \mathbf{r})=1 /\left(16 \pi^{2}|\mathbf{x}-\mathbf{s}||\mathbf{x}-\mathbf{r}|\right)$ and $T(\mathbf{r}, \mathbf{x}, \mathbf{s})=T(\mathbf{x}, \mathbf{r})+$ $T(\mathbf{s}, \mathbf{x})$. The integration in $t$ results in $2 \pi \delta\left(\omega-\omega^{\prime}\right)$. Performing both this integration and that in $\omega^{\prime}$ results in

$$
\begin{aligned}
& N[\delta c](\mathbf{y})=\frac{1}{2 \pi} \int_{\mathbb{R}} \mathrm{d} \omega \int_{O_{r} \times O_{s}} \int_{X}\left[\omega^{4} 4 c^{-6} A(\mathbf{s}, \mathbf{x}, \mathbf{r})\right. \\
& \left.\quad \times A(\mathbf{s}, \mathbf{y}, \mathbf{r}) e^{i \omega(T(\mathbf{r}, \mathbf{x}, \mathbf{s})-T(\mathbf{r}, \mathbf{y}, \mathbf{s}))}\right] \delta c(\mathbf{x}) \mathrm{d} \mathbf{x} \mathrm{d} \mathbf{s} \mathrm{d} \mathbf{r} .
\end{aligned}
$$

We now change variables to the ray directions at the scattering point ( $\boldsymbol{\alpha}^{s}$ and $\boldsymbol{\alpha}^{r}$ ). We will use subscripts 1, 2, 3 to denote the components of a vector in the $x_{1}, x_{2}, x_{3}$ direction, respectively. To change from $(\mathbf{s}, \mathbf{r})$ to $\left(\boldsymbol{\alpha}^{s}, \boldsymbol{\alpha}^{r}\right)$, we compute the Jacobian:

$$
\frac{\partial\left(\alpha_{1}^{s}, \alpha_{2}^{s}\right)}{\partial\left(s_{1}, s_{2}\right)} \frac{\partial\left(\alpha_{1}^{r}, \alpha_{2}^{r}\right)}{\partial\left(r_{1}, r_{2}\right)}=\frac{y_{3}^{2}}{|\mathbf{y}-\mathbf{s}|^{4}} \frac{y_{3}^{2}}{|\mathbf{y}-\mathbf{r}|^{4}}
$$

since

$$
\alpha_{1,2}^{s}=\frac{y_{1,2}-s_{1,2}}{|\mathbf{y}-\mathbf{s}|} \text { and } \quad \alpha_{1,2}^{r}=\frac{y_{1,2}-r_{1,2}}{|\mathbf{y}-\mathbf{r}|}
$$

Performing this change of variables, we obtain

$$
\begin{aligned}
N & {[\delta c](\mathbf{y}) \simeq \frac{1}{2 \pi} \int_{\mathbb{R}} \mathrm{d} \omega \int_{S_{s}^{2} \times S_{r}^{2}} \int_{X}\left[\omega^{4} 4 c^{-6} A(\underline{\mathbf{s}}, \mathbf{y}, \underline{\mathbf{r}})^{2}\right.} \\
& \left.\times \frac{|\mathbf{y}-\underline{\mathbf{s}}|^{4}|\mathbf{y}-\underline{\mathbf{r}}|^{4}}{y_{3}^{4}} e^{i \omega(T(\underline{\mathbf{r}}, \mathbf{x}, \underline{\mathbf{s}})-T(\underline{\mathbf{r}}, \mathbf{y}, \underline{\mathbf{s}}))}\right] \\
& \times \delta c(\mathbf{x}) \mathrm{d} \mathbf{x} \mathrm{d} \boldsymbol{\alpha}^{s} \mathrm{~d} \boldsymbol{\alpha}^{r} .
\end{aligned}
$$

Underlined symbols indicate a variable is a function of other variables and $S_{s}^{2}\left(S_{r}^{2}\right)$ is the unit sphere on which the direction of the source (receiver) ray lies. We have expanded $A(\underline{\mathbf{s}}, \mathbf{x}, \underline{\mathbf{r}})$ about $\mathbf{y}$, using that $\mathbf{x} \approx \mathbf{y}$ at stationarity in $\boldsymbol{\alpha}^{r}$ and $\boldsymbol{\alpha}^{s}$ [see de Hoop and Brandsberg-Dahl (2000, p. 553) and Beylkin (1985)].

Expanding $T(\underline{\mathbf{r}}, \mathbf{x}, \underline{\mathbf{s}})$ to first order in a Taylor series about $\mathbf{y}$, we can write the phase as

$$
\Gamma \cdot(\mathbf{x}-\mathbf{y})
$$

where $\Gamma=\nabla_{\mathbf{y}} T(\underline{\mathbf{r}}, \mathbf{y}, \underline{\mathbf{s}})$. We also scale the $\omega$ variable by $|\Gamma|^{-1}$ and introduce $\nu=\Gamma /|\Gamma|$. Note that $\nu$ corresponds to the migration dip. The expression thus becomes

$$
\begin{aligned}
N[\delta c](\mathbf{y}) \simeq & \frac{1}{2 \pi} \int_{\mathbb{R}} \mathrm{d} \omega \int_{S_{s}^{2} \times S_{r}^{2}} \int_{X} \\
& {\left[4 c^{-6} \omega^{4} \mu_{L S}\left(\underline{\mathbf{r}}, \boldsymbol{\alpha}^{r}, \underline{\mathbf{s}}, \boldsymbol{\alpha}^{s}, \mathbf{y}\right) e^{i \omega \boldsymbol{\nu} \cdot(\mathbf{x}-\mathbf{y})}\right] } \\
& \times \delta c(\mathbf{x}) \mathrm{d} \mathbf{x} \mathrm{d} \boldsymbol{\alpha}^{s} \mathrm{~d} \boldsymbol{\alpha}^{r},
\end{aligned}
$$

letting

$$
\mu_{L S}\left(\underline{\mathbf{r}}, \boldsymbol{\alpha}^{r}, \underline{\mathbf{s}}, \boldsymbol{\alpha}^{s}, \mathbf{y}\right)=A(\underline{\mathbf{s}}, \mathbf{y}, \underline{\mathbf{r}})^{2} \frac{\partial(\mathbf{s}, \mathbf{r})}{\partial\left(\boldsymbol{\alpha}^{s}, \boldsymbol{\alpha}^{r}\right)}|\boldsymbol{\Gamma}|^{-5} .
$$

We now change variables again to the scattering angle $\theta$, scattering azimuth $\psi$, and migration dip $\boldsymbol{\nu}$ [these quantities are defined in terms of $\boldsymbol{\alpha}^{s}$ and $\boldsymbol{\alpha}^{r}$ by de Hoop and BrandsbergDahl (2000, equation 127)]. This introduces a Jacobian, $\partial\left(\boldsymbol{\alpha}^{s}, \boldsymbol{\alpha}^{s}\right) / \partial(\boldsymbol{\nu}, \theta, \psi)$, which is computed for the general case by Burridge et al. (1998) and for the homogeneous, isotropic case by Burridge and Beylkin (1988). In a 3D (homogeneous and isotropic) space, this Jacobian is $\sin \theta$. Thus,

$$
\begin{aligned}
N[\delta c](\mathbf{y}) \simeq & \frac{1}{2 \pi} \int_{\mathbb{R}} \mathrm{d} \omega \int_{E_{v}} \int_{E_{\theta} \times E_{\psi}} \int_{X} \\
& {\left[4 c^{-6} \omega^{4} \mu_{L S}\left(\underline{\mathbf{r}}, \underline{\boldsymbol{\alpha}}^{r}, \underline{\mathbf{s}}, \underline{\boldsymbol{\alpha}}^{s}, \mathbf{y}\right) \sin \theta e^{i \omega \boldsymbol{\nu} \cdot(\mathbf{y}-\mathbf{x})}\right] } \\
& \times \delta c(\mathbf{x}) \mathrm{d} \mathbf{x} \mathrm{d} \boldsymbol{\nu} \mathrm{d} \theta \mathrm{d} \psi
\end{aligned}
$$

where we change the sign of $\omega$, noting that $\int_{\mathbb{R}} e^{i \omega(\mathbf{x}-\mathbf{y})} \mathrm{d} \omega=$ $\int_{\mathbb{R}} e^{i \omega(\mathbf{y}-\mathbf{x})} \mathrm{d} \omega$ and that the amplitude is an even function of $\omega$. We denote by $E_{v}$ the set of migration dips, by $E_{\theta}$ the set of scattering angles and by $E_{\psi}$ the set of scattering azimuths.

Letting $\Lambda(\mathbf{y}, \boldsymbol{\nu})=\int_{E_{\theta} \times E_{\psi}} \frac{1}{2} \mu_{L S}\left(\underline{\mathbf{r}}, \underline{\boldsymbol{\alpha}}^{r}, \underline{\mathbf{s}}, \underline{\boldsymbol{\alpha}}^{s}, \mathbf{y}\right) \sin \theta \mathrm{d} \psi \mathrm{d} \theta+$ $(\ldots)(\mathbf{y},-\boldsymbol{\nu})[(\ldots)(\mathbf{y},-\boldsymbol{\nu})$ indicates the same expression evaluated at $(\mathbf{y},-\boldsymbol{\nu})$, noting that $\boldsymbol{\alpha}^{r}$ and $\boldsymbol{\alpha}^{s}$ are related to $\left.\boldsymbol{\nu}\right]$, we 
may write

$$
\begin{aligned}
N[\delta c](\mathbf{y}) \simeq & \left(\frac{1}{2 \pi}\right)^{3} \int_{\mathbb{R}_{+}} \omega^{2} \mathrm{~d} \omega \int_{E_{\nu}} \omega^{2} 4 c^{-6} 8 \pi^{2} \Lambda(\mathbf{y}, \boldsymbol{\nu}) \\
& \times \int_{X} e^{i \omega \nu \cdot(\mathbf{y}-\mathbf{x})} \delta c(\mathbf{x}) \mathrm{d} \mathbf{x} \mathrm{d} \boldsymbol{\nu}
\end{aligned}
$$

from which we recognize $\omega^{2} \mathrm{~d} \omega \mathrm{d} \nu$ as a measure of integration in spherical coordinates, where $\omega$ takes the place of radial length and $\nu_{1,2}$ of directions. We see also that $\omega^{2} 4 c^{-6} 8 \pi^{2}$ $\Lambda(\mathbf{y}, \nu)$ is the principal symbol (Treves, 1980a; de Hoop et al., 2003a, Appendix A) of the normal operator, the highest order term in the asymptotic expansion of its amplitude in the Fourier domain. Since we associate $\omega$ with a length, the $\omega$ integration should be over only $\mathbb{R}_{+}$. By noting that the argument is an even function of $\omega$, we replace the integration over $\mathbb{R}$ with integration over $\mathbb{R}_{+}$introducing a factor of two. The remaining $\omega^{2}$ term is the (scaled) second derivative operator. The kernel of the parametrix (Treves 1980a; de Hoop et al., 2003a, Appendix A), or the asymptotic approximatation to the inverse, of the normal operator is

$$
\begin{aligned}
P(\mathbf{x}, \mathbf{y}) \simeq & \left(\frac{1}{2 \pi}\right)^{3} \int_{\mathbb{R}_{+}} \int_{E_{v}}\left(4 c^{-6} 8 \pi^{2} \Lambda(\mathbf{y}, \nu)\right)^{-1} \\
& \times e^{i \omega \boldsymbol{\nu} \cdot(\mathbf{x}-\mathbf{y})} \mathrm{d} \boldsymbol{\nu} \mathrm{d} \omega .
\end{aligned}
$$

To construct the linear inversion operator, we compose $P$ with the original imaging operator to form $\left(F^{*} F\right)^{-1} F^{*}$ :

$$
\begin{aligned}
& P F^{*}[u](\mathbf{y})=\left(\frac{1}{2 \pi}\right)^{4} \int_{E_{v}} \int_{X} \int_{\mathbb{R}_{+}} \int_{O_{r} \times O_{s}} \\
& A(\mathbf{s}, \mathbf{x}, \mathbf{r}) 2 c^{-3}\left(4 c^{-6} 8 \pi^{2} \Lambda(\mathbf{x}, \boldsymbol{\nu})\right)^{-1} e^{i \omega \boldsymbol{\nu} \cdot(\mathbf{x}-\mathbf{y})} \\
& \times \int_{\mathbb{R}}\left(-\omega^{\prime 2}\right) e^{-i \omega^{\prime} T(\mathbf{r}, \mathbf{x}, \mathbf{s})} u\left(\mathbf{r}, \mathbf{s}, \omega^{\prime}\right) \mathrm{d} \omega^{\prime} \mathrm{d} \mathbf{s} \mathrm{d} \mathbf{r} \mathrm{d} \omega \mathrm{d} \mathbf{x} \mathrm{d} \boldsymbol{\nu}
\end{aligned}
$$

where the data $u$ are now in the frequency domain. By expanding $T(\mathbf{r}, \mathbf{x}, \mathbf{s})$ to first order in a Taylor series about $\mathbf{x}=\mathbf{y}$, we can write the phase of the above operator as

$$
i \omega \nu \cdot(\mathbf{x}-\mathbf{y})-i \omega^{\prime} \nabla_{y} T(\mathbf{r}, \mathbf{y}, \mathbf{s}) \cdot(\mathbf{x}-\mathbf{y})-i \omega^{\prime} T(\mathbf{r}, \mathbf{y}, \mathbf{s}) .
$$

We can now perform the integration in $\mathbf{x}$ (noting that $\mathbf{x} \approx \mathbf{y}$ at stationarity), giving

$$
\begin{aligned}
& (2 \pi)^{3} \delta\left(\omega \nu-\omega^{\prime} \nabla_{y} T(\mathbf{r}, \mathbf{y}, \mathbf{s})\right) \\
& \quad=\frac{(2 \pi)^{3}}{\omega^{\prime 2}|\boldsymbol{\Gamma}|^{2}} \delta\left(\omega-\omega^{\prime}|\boldsymbol{\Gamma}|\right) \delta(\nu-\mathbf{v}),
\end{aligned}
$$

where $\mathbf{v}=\nabla_{y} T(\mathbf{r}, \mathbf{y}, \mathbf{s}) /\left|\nabla_{y} T(\mathbf{r}, \mathbf{y}, \mathbf{s})\right|$. This allows us to integrate out both the $\nu$ and $\omega$ variables, resulting in

$$
\begin{array}{r}
P F^{*}[u](\mathbf{y})=\frac{1}{8 \pi^{2}} \int_{O_{r} \times O_{s}} \frac{1}{|\boldsymbol{\Gamma}|^{2}}(\Lambda(\mathbf{y}, \mathbf{v}))^{-1} \frac{1}{2} c^{3} A(\mathbf{s}, \mathbf{y}, \mathbf{r}) \\
\times \frac{1}{\pi} \operatorname{Re}\left[\int_{\mathbb{R}_{+}} u\left(\mathbf{s}, \mathbf{r}, \omega^{\prime}\right) e^{-i \omega^{\prime} T(\mathbf{r}, \mathbf{y}, \mathbf{s})} \mathrm{d} \omega^{\prime}\right] \mathrm{d} \mathbf{s} \mathrm{d} \mathbf{r} . \quad(\mathrm{B}-17)
\end{array}
$$

Changing variables from $(\mathbf{s}, \mathbf{r})$ to $\left(\boldsymbol{\alpha}^{s}, \boldsymbol{\alpha}^{r}\right)$ as before and substituting $\mu_{L S}\left(\underline{\mathbf{r}}, \boldsymbol{\alpha}^{r}, \underline{\mathbf{s}}, \boldsymbol{\alpha}^{s}, \mathbf{y}\right)$ gives

$$
\begin{aligned}
& I[u](\mathbf{y})=P F^{*}[u](\mathbf{y}) \\
& \quad=\frac{1}{16 \pi^{2}} \int_{S_{s}^{2} \times S_{r}^{2}} \frac{c^{3}|\boldsymbol{\Gamma}|^{3}(\Lambda(\mathbf{y}, \mathbf{v}))^{-1} \mu_{L S}\left(\underline{\mathbf{r}}, \boldsymbol{\alpha}^{r}, \underline{\mathbf{s}}, \boldsymbol{\alpha}^{s}, \mathbf{y}\right)}{A(\underline{\mathbf{s}}, \mathbf{y}, \underline{\mathbf{r}})} \\
& \quad \times \frac{1}{\pi} \operatorname{Re}\left[\int_{\mathbb{R}_{+}} e^{-i \omega^{\prime} T(\underline{\mathbf{r}}, \mathbf{y}, \underline{\mathbf{s}})} u\left(\underline{\mathbf{s}}, \underline{\mathbf{r}}, \omega^{\prime}\right) \mathrm{d} \omega^{\prime}\right] \mathrm{d} \boldsymbol{\alpha}^{s} \mathrm{~d} \boldsymbol{\alpha}^{r} .
\end{aligned}
$$

We can then perform the integration with respect to $\omega^{\prime}$, obtaining

$$
\begin{aligned}
& I[u](\mathbf{y}) \\
& \quad=\frac{1}{16 \pi^{2}} \int_{S_{s}^{2} \times S_{r}^{2}} \frac{c^{3}|\boldsymbol{\Gamma}|^{3}(\Lambda(\mathbf{y}, \mathbf{v}))^{-1} \mu_{L S}\left(\underline{\mathbf{r}}, \boldsymbol{\alpha}^{r}, \underline{\mathbf{s}}, \boldsymbol{\alpha}^{s}, \mathbf{y}\right)}{A(\underline{\mathbf{s}}, \mathbf{y}, \underline{\mathbf{r}})} \\
& \quad \times u(\underline{\mathbf{s}}, \underline{\mathbf{r}}, T(\underline{\mathbf{r}}, \mathbf{y}, \underline{\mathbf{s}})) \mathrm{d} \boldsymbol{\alpha}^{s} \mathrm{~d} \boldsymbol{\alpha}^{r} .
\end{aligned}
$$

In equation B-18, we recognize the form of equation 27 of Miller et al. (1987) by noting that

$$
|\boldsymbol{\Gamma}|=\frac{2 \cos \left(\frac{\theta}{2}\right)}{c}
$$

and setting $\mu_{L S}=1$. The two equations are not exactly the same, however; our equation contains a factor $\sin \theta / \Lambda$. The $\sin \theta$ is introduced by changing to the same coordinates as used by Miller et al. (1987). The factor $\Lambda^{-1}$ is present since we construct the least-squares inverse; it is equal to $\int_{E_{\theta}} \sin \theta \mathrm{d} \theta$.

\section{Continuation}

To compute the continuation operator, we now compose $I$ with the modeling operator:

$$
\begin{aligned}
F I & {[u](\mathbf{s}, \mathbf{r}, t)=\frac{1}{2 \pi} \int_{\mathbb{R}} \int_{X} \frac{1}{16 \pi^{2}} \int_{S_{s}^{2} \times S_{r}^{2}} \frac{1}{2 \pi} } \\
& \times \int_{\mathbb{R}}|\boldsymbol{\Gamma}|^{3}(\Lambda(\mathbf{x}, \mathbf{v}))^{-1} \frac{-\omega^{2} A(\mathbf{s}, \mathbf{x}, \mathbf{r})}{A\left(\underline{\mathbf{s}}^{\prime}, \mathbf{x}, \underline{\mathbf{r}}^{\prime}\right)} \\
& \times \mu_{L S}\left(\underline{\mathbf{r}}^{\prime}, \boldsymbol{\alpha}^{r \prime}, \underline{\mathbf{s}}^{\prime}, \boldsymbol{\alpha}^{s \prime}, \mathbf{x}\right) e^{-i \omega(t-T(\mathbf{r}, \mathbf{x}, \mathbf{s}))-i \omega^{\prime} T\left(\underline{\mathbf{r}}^{\prime}, \mathbf{x}, \underline{\mathbf{s}}^{\prime}\right)} \\
& \times u\left(\underline{\mathbf{s}}^{\prime}, \underline{\mathbf{r}}^{\prime}, \omega^{\prime}\right) \mathrm{d} \omega^{\prime} \mathrm{d} \boldsymbol{\alpha}^{s^{\prime}} \mathrm{d} \boldsymbol{\alpha}^{r \prime} \mathrm{d} \mathbf{x} \mathrm{d} \omega,
\end{aligned}
$$

or, in $\left(\theta^{\prime}, \psi^{\prime}, \nu^{\prime}\right)$ variables, exchanging the order of integration,

$$
\begin{aligned}
& F I[u](\mathbf{s}, \mathbf{r}, t)=\frac{1}{2 \pi} \int_{\mathbb{R}} \frac{1}{16 \pi^{2}} \int_{\nu^{\prime}} \int_{E_{\theta^{\prime}} \times E_{\psi^{\prime}}} \int_{X} \frac{1}{2 \pi} \\
& \times \int_{\mathbb{R}} \sin \theta^{\prime}|\boldsymbol{\Gamma}|^{3}(\Lambda(\mathbf{x}, \mathbf{v}))^{-1} \frac{-\omega^{2} A(\mathbf{s}, \mathbf{x}, \mathbf{r})}{A\left(\underline{\mathbf{s}}^{\prime}, \mathbf{x}, \underline{\mathbf{r}}^{\prime}\right)} \\
& \times \mu_{L S}\left(\underline{\mathbf{r}}^{\prime}, \underline{\boldsymbol{\alpha}}^{r \prime}, \underline{\mathbf{s}}^{\prime}, \underline{\boldsymbol{\alpha}}^{S^{\prime}}, \mathbf{x}\right) e^{-i \omega(t-T(\mathbf{r}, \mathbf{x}, \mathbf{s}))-i \omega^{\prime} T\left(\underline{\mathbf{r}}^{\prime}, \mathbf{x}, \underline{\mathbf{s}}^{\prime}\right)} \\
& \times u\left(\underline{\mathbf{s}}^{\prime}, \underline{\mathbf{r}}^{\prime}, \omega^{\prime}\right) \mathrm{d} \omega^{\prime} \mathrm{d} \mathbf{x} \mathrm{d} \nu^{\prime} \mathrm{d} \theta^{\prime} \mathrm{d} \psi^{\prime} \mathrm{d} \omega .
\end{aligned}
$$

We now approximate the $\mathbf{x}$-integration using the method of stationary phase. To do this, we first change variables to 
$\left(\tau^{\prime}, \sigma_{1}^{\prime}, \sigma_{2}^{\prime}\right)$, where $\left(\sigma_{2}^{\prime}, \sigma_{2}^{\prime}\right)$ are coordinates on the level sets of $T\left(\underline{\mathbf{r}}^{\prime}, \mathbf{x}, \underline{\mathbf{s}}^{\prime}\right)$ and $\tau^{\prime}$ is the value of $T\left(\underline{\mathbf{r}}^{\prime}, \mathbf{x}, \underline{\mathbf{s}}^{\prime}\right)$, which fixes the level set on which we perform the computations. This transformation is accomplished by noting that

$$
\mathrm{d} \mathbf{x}=\frac{\left|\partial_{\sigma_{1}^{\prime}} \underline{\mathbf{x}} \wedge \partial_{\sigma_{2}^{\prime}} \underline{\mathbf{x}}\right|}{\left|\nabla_{\underline{x}} T^{\prime}\right|} \mathrm{d} \tau^{\prime} \mathrm{d} \sigma_{1}^{\prime} \mathrm{d} \sigma_{2}^{\prime}
$$

(see de Hoop and Bleistein, 1997) where $T^{\prime}=T\left(\mathbf{r}^{\prime}, \mathbf{x}, \mathbf{s}^{\prime}\right)$.

If $\underline{\mathbf{r}}^{\prime}$ and $\underline{\mathbf{s}}^{\prime}$ were independent parameters, then the level sets of $T^{\prime}$ would define standard isochrons and the stationary phase analysis could be found in Bleistein et al. (2000). If instead we want the level sets of $T^{\prime}$ to be the angle isochrons shown in the text of this paper, we must change our variables of integration from $\left(\nu^{\prime}, \psi^{\prime}, \theta^{\prime}\right)$ to $\left(\mathbf{s}^{\prime}, \psi^{\prime}\right.$, $\left.\theta^{\prime}\right)$, which introduces the Jacobian $\partial\left(\boldsymbol{\nu}^{\prime}, \boldsymbol{\psi}^{\prime}, \theta^{\prime}\right) / \partial\left(\mathbf{s}^{\prime}, \boldsymbol{\psi}^{\prime}, \theta^{\prime}\right)$. In this case, $\mathbf{s}^{\prime}$ is an independent parameter but $\underline{\mathbf{r}}^{\prime}$ is no longer independent since

$$
\underline{\mathbf{r}}^{\prime}\left(\mathbf{x}, \mathbf{s}^{\prime}, \theta^{\prime}, \psi^{\prime}\right)=\mathbf{x}-\left(\frac{\underline{x}_{3}}{\underline{\alpha}_{3}^{\prime}}\right) \underline{\boldsymbol{\alpha}}^{r^{\prime}},
$$

using the definitions of $\underline{\psi}^{\prime}$ and $\underline{\boldsymbol{\alpha}}^{\prime}$ from de Hoop and Brandsberg-Dahl (2000):

$$
\begin{aligned}
\underline{\boldsymbol{\alpha}}^{r \prime} & =\frac{\mathbf{x}-\mathbf{s}^{\prime}}{\left|\mathbf{x}-\mathbf{s}^{\prime}\right|}-\frac{\psi^{\prime} \cos \left(\frac{\theta^{\prime}}{2}\right)}{\sin \theta^{\prime}}, \\
\underline{\boldsymbol{\psi}}^{\prime} & =\frac{\left(\underline{\boldsymbol{\alpha}}^{s^{\prime}} \cdot \boldsymbol{\nu}^{\prime}\right) \underline{\boldsymbol{\alpha}}^{r \prime}-\left(\underline{\boldsymbol{\alpha}}^{r \prime} \cdot \boldsymbol{\nu}^{\prime}\right) \underline{\boldsymbol{\alpha}}^{s^{\prime}}}{\sin \theta^{\prime}} .
\end{aligned}
$$

In this parameterization $D_{x_{j}} T^{\prime}=\partial_{x_{j}} T^{\prime}+\partial_{r_{i}^{\prime}} T^{\prime} \partial_{x_{j}} \underline{r}_{i}^{\prime}$ because of the $\mathbf{x}$-dependence of $\underline{\mathbf{r}}^{\prime}$. In the isochron variables, the phase of the continuation operator becomes

$$
\Phi=T\left(\mathbf{r}, \underline{\mathbf{x}}\left(\sigma_{1}^{\prime}, \sigma_{2}^{\prime}, \tau^{\prime}\right), \mathbf{s}\right)-t-\frac{\omega^{\prime}}{\omega} \tau^{\prime} .
$$

We now continue with the stationary phase analysis in $\left(\sigma_{1}^{\prime}, \sigma_{2}^{\prime}\right)$, following Bleistein et al. (2000). From the stationary phase condition

$$
\partial_{\sigma_{1,2}^{\prime}} \Phi=\nabla_{\underline{x}} T \cdot \frac{\partial \underline{\mathbf{x}}}{\partial \sigma_{1,2}^{\prime}}=0
$$

it follows that $\nabla_{\underline{x}} T$ is parallel to $\nabla_{\underline{x}} T^{\prime}$ or equivalently, the unit normal to the input angle isochron $\hat{n}^{\prime}$ is equal to the unit normal $\hat{n}$ to the output standard isochron. The Hessian of second derivatives is

$$
\mathbf{H}=\operatorname{det}\left[\frac{\partial^{2} T}{\partial \sigma_{1}^{\prime} \partial \sigma_{2}^{\prime}}\right]=\left|\nabla_{\underline{x}} T\right|^{2} \operatorname{det}\left[\mathbf{B}_{k l}^{\prime}-\mathbf{B}_{k l}\right],
$$

where we use the relation

$$
\frac{\partial^{2} T}{\partial \sigma_{l}^{\prime} \partial \sigma_{k}^{\prime}}=\frac{\partial^{2} T}{\partial \underline{x}_{i} \partial \underline{x}_{j}} \frac{\partial \underline{x}_{i}}{\partial \sigma_{k}^{\prime}} \frac{\partial \underline{x}_{j}}{\partial \sigma_{l}^{\prime}}+\frac{\partial T}{\partial \underline{x}_{i}} \frac{\partial^{2} \underline{x}_{i}}{\partial \sigma_{k}^{\prime} \partial \sigma_{l}^{\prime}}
$$

and

$$
\frac{\partial T}{\partial \underline{x}_{i}} \frac{\partial^{2} \underline{x}_{i}}{\partial \sigma_{k}^{\prime} \partial \sigma_{l}^{\prime}}=\hat{\mathbf{n}} \cdot \frac{\partial^{2} \underline{x}}{\partial \sigma_{k}^{\prime} \partial \sigma_{l}^{\prime}}\left|\nabla_{\underline{x}} T\right|=\mathbf{B}_{k l}^{\prime}\left|\nabla_{\underline{x}} T\left(\underline{\mathbf{r}^{\prime}}, \underline{\mathbf{x}}, \mathbf{s}^{\prime}\right)\right|,
$$

using the fact that $\hat{\mathbf{n}}^{\prime}=\hat{\mathbf{n}}$ at stationarity. The second fundamental tensor, $\mathbf{B}_{k l}$ is a rotation of the matrix of principle curvatures for the level sets (standard isochrons) of the function $T$. The term $\mathbf{B}_{k l}^{\prime}$ denotes $\mathbf{B}_{k l}$ in the input parameters [i.e., a rotation of the matrix of principle curvatures for the level sets (angle isochrons) of $\left.T^{\prime}\right]$. Equation B-26 gives $\mathbf{B}_{k l}^{\prime}$ because $\left(\sigma_{1}^{\prime}\right.$, $\left.\sigma_{2}^{\prime}\right)$ are coordinates on the angle isochrons (level sets of $T^{\prime}$ ). The transformation of the first term in equation B-25 to $\mathbf{B}_{k l}$ is explained in Bleistein et al. (2000, section 7.7).

The preceding stationary phase analysis is applicable only when the Hessian $\mathbf{H}$ is nonzero. One example of when $\mathbf{H}=0$ is when the input and output configurations are the same, in which case $\mathbf{B}_{k l}=\mathbf{B}_{k l}^{\prime}$. Assuming that $\mathbf{H} \neq 0$, we can write the final continuation operator as

$$
\begin{aligned}
& F I[u](\mathbf{s}, \mathbf{r}, t)=\int_{E_{\theta^{\prime}} \times E_{\psi^{\prime}}} \frac{-1}{2 \pi} \int_{\mathbb{R}_{+}} \int_{\mathbb{R}} \frac{1}{4 \sqrt{2 \pi}} \\
& \times \int_{S^{\prime}} \omega \sin \theta^{\prime}|\boldsymbol{\Gamma}|^{3}\left(\Lambda\left(\underline{\mathbf{x}}_{0}, \mathbf{v}\right)\right)^{-1} \mu_{L S}\left(\underline{\mathbf{r}}^{\prime}, \underline{\boldsymbol{\alpha}}^{r \prime}, \mathbf{s}^{\prime}, \underline{\boldsymbol{\alpha}}^{s^{\prime}}, \underline{\mathbf{x}}_{0}\right) \\
& \quad \times\left|\partial_{\sigma_{1}} \underline{\mathbf{x}} \wedge \partial_{\sigma_{2}} \underline{\mathbf{x}}\right| \frac{\partial\left(\boldsymbol{\nu}^{\prime}, \psi^{\prime}, \theta^{\prime}\right)}{\partial\left(\mathbf{s}^{\prime}, \psi^{\prime}, \theta^{\prime}\right)} \mid \frac{A\left(\mathbf{s}, \underline{\mathbf{x}}_{0}, \mathbf{r}\right)}{A\left(\mathbf{s}^{\prime}, \underline{\mathbf{x}}_{0}, \underline{\mathbf{r}}^{\prime}\right) \sqrt{\mathbf{H}}\left|\nabla_{x_{0}} T^{\prime}\right|} \\
& \quad \times u\left(\mathbf{s}^{\prime}, \underline{\mathbf{r}}^{\prime}, T\left(\underline{\mathbf{r}}^{\prime}, \underline{\mathbf{x}}_{0}, \mathbf{s}^{\prime}\right)\right) e^{i \omega\left(T\left(\mathbf{r}, \underline{\mathbf{x}}_{0}, \mathbf{s}\right)-t\right)+i \frac{\pi}{4} \operatorname{sig}(\mathbf{H})} \\
& \quad \times \mathrm{d} \mathbf{s}^{\prime} \mathrm{d} \omega \mathrm{d} \tau^{\prime} \mathrm{d} \theta^{\prime} \mathrm{d} \psi^{\prime} .
\end{aligned}
$$

In this expression, $\mathbf{x}_{0}$ is the stationary point [in $\sigma_{1}^{\prime}$ and $\sigma_{2}^{\prime}$, i.e., $\left.\underline{\mathbf{x}}_{0}=\underline{x}\left(\sigma_{1}^{\prime 0}, \sigma_{2}^{\prime 0}, \tau^{\prime}\right), \nabla_{\underline{x}_{0}} T^{\prime}=\left.\nabla_{\underline{x}} T\left(\underline{\mathbf{r}}^{\prime}, \underline{\mathbf{x}}, \mathbf{s}^{\prime}\right)\right|_{\underline{x}=\underline{x}_{0}}\right]$ and $\operatorname{sig}(\mathbf{H})$ is not evaluated as this depends on the exact input and output configuration.

Equation B-27 can be reduced to equation 7.7.13 of Bleistein et al. (2002) by setting $\mu_{L S}=1$ and noting that $|\Gamma|^{3}$ is the obliquity factor. This comparison is possible only after noting the differences between the definitions of the modeling and inversion operators between this work and that of Bleistein et al. (2002). As in comparing the inversion formula with that of Miller et al. (1987), the final two formulas differ by a factor $\sin \theta^{\prime} / \int E_{\theta} \sin \theta^{\prime}$.

Other authors have done similar computations to that shown here for particular input and output geometries. For example, Biondi et al. (1998) compute the operator that maps general input data to single-azimuth output data; Black et al. (1993), Liner (1991), and Bleistein (1990) compute the DMO operator; and more general continuation is given in Bleistein et al. (1999), Bleistein and Jaramillo (2000), Fomel and Bleistein (2001), Stolt (2002), and Fomel (2003).

\section{APPENDIX C}

\section{PROPERTIES OF FF $^{*}$ FOR COMMON OFFSET}

Because formal definition of a pseudodifferential operator is beyond the scope of this paper, we refer the reader to either Treves' work (1980a) or Appendix A of de Hoop et al. (2003a) for the mathematical details. Roughly speaking, an operator is pseudodifferential if it can be written in the form of a forward and inverse Fourier transform along with a multiplication by an amplitude in the Fourier domain. In general, $F F^{*}$ is not a pseudodifferential operator. In certain cases, however, such as 
when the input and output offsets are constrained to be equal, $F F^{*}$ does have this property. In this particular case, we write the kernel of $F F^{*}$ as that of a pseudodifferential operator and extract its amplitude behavior.

The phase of $F F^{*}$ is given by (see equation B-20)

$$
\Phi=-\omega(t-T(\mathbf{r}, \mathbf{x}, \mathbf{s}))+\omega^{\prime}\left(t^{\prime}-T\left(\mathbf{r}^{\prime}, \mathbf{x}, \mathbf{s}^{\prime}\right)\right),
$$

in which $\mathbf{x}, \omega$, and $\omega^{\prime}$ are identified as phase variables. Subjecting equation $\mathrm{C}-1$ to the common offset condition, the traveltime $T$ and phase function $\Phi$ can be rewritten in terms of midpoints $\mathbf{y}=(\mathbf{s}+\mathbf{r}) / 2, \mathbf{y}^{\prime}=\left(\mathbf{s}^{\prime}+\mathbf{r}^{\prime}\right) / 2$ and offset $\mathbf{h}(\mathbf{s}-\mathbf{r}) / 2$ $=\left(\mathbf{s}^{\prime}-\mathbf{r}^{\prime}\right) / 2$, as

$$
\Phi=-\omega(t-T(\mathbf{x}, \mathbf{h}, \mathbf{y}))+\omega^{\prime}\left(t^{\prime}-T\left(\mathbf{x}, \mathbf{h}, \mathbf{y}^{\prime}\right)\right) .
$$

\section{Treatment of phase variables}

The representation of the kernel of the operator $F F^{*}$ contains integrations over $\mathbf{x}, \omega$, and $\omega^{\prime}$. The application of this operator to input data results in integrations over $\mathbf{y}^{\prime}$ and $t^{\prime}$, the input midpoint and time (but not over $\mathbf{h}$ as this is fixed). For this phase to be that of a pseudodifferential operator, we must be able to write it in the form

$$
\omega^{\prime}\left(t^{\prime}-t\right)+\boldsymbol{\eta}^{\prime} \cdot\left(\mathbf{y}-\mathbf{y}^{\prime}\right)
$$

where $\omega^{\prime} t^{\prime}$ and $-\boldsymbol{\eta}^{\prime} \cdot \mathbf{y}^{\prime}$ are associated with Fourier transforms and $-\omega^{\prime} t$ and $\boldsymbol{\eta}^{\prime} \cdot \mathbf{y}$ are associated with inverse Fourier transforms. Thus, the oscillatory integral representation of the kernel of $F F^{*}$ must contain integrations over $\left(\omega^{\prime}, \boldsymbol{\eta}^{\prime}\right)$, the Fourier duals of $\left(t^{\prime}, \mathbf{y}^{\prime}\right)$. To reduce the phase C-2 to the form in equation C-3, we apply the method of stationary phase to $\left(x_{3}, \omega\right)$ and then change variables of integration: $\left(x_{1}, x_{2}\right) \rightarrow\left(\eta_{1}, \eta_{2}\right)$.

To begin, we perform stationary phase in $\left(x_{3}, \omega\right)$, the depth and the output frequency. The stationary phase conditions are

$$
\begin{aligned}
& \frac{\partial \Phi}{\partial \omega}=T(\mathbf{x}, \mathbf{h}, \mathbf{y})-t=0, \\
& \frac{\partial \Phi}{\partial x_{3}}=\omega \partial_{x_{3}} T(\mathbf{x}, \mathbf{h}, \mathbf{y})-\omega^{\prime} \partial_{x_{3}} T\left(\mathbf{x}, \mathbf{h}, \mathbf{y}^{\prime}\right)=0 .
\end{aligned}
$$

Equation C-4 determines the isochron on which the scattering point $\mathbf{x}$ lies through its solution $\underline{x}_{3}^{0}\left(x_{1}, x_{2}, \mathbf{y}, t ; \mathbf{h}\right)$, equation C-5 gives the scaling between the input and output frequencies at stationarity through its solution

$$
\underline{\omega}^{0}\left(\omega^{\prime}, x_{1}, x_{2}, \mathbf{y}, \mathbf{y}^{\prime}, h\right)=\omega^{\prime} \frac{\partial_{x_{3}} T\left(\mathbf{x}, \mathbf{h}, \mathbf{y}^{\prime}\right)}{\partial_{x_{3}} T(\mathbf{x}, \mathbf{h}, \mathbf{y})} .
$$

The determinant of the Hessian $\mathbf{H}$ is

$$
-\left(\partial_{x_{3}} T(\mathbf{x}, \mathbf{h}, \mathbf{y})\right)^{2},
$$

which is unequal to zero since we exclude the direct ray between the source and receiver (i.e., we require $x_{3} \neq 0$ in a constant medium). Since this $2 \times 2$ determinant is negative, $\operatorname{sig}(\mathbf{H})=0$.

The phase at stationarity can be written as

$$
\begin{aligned}
\Phi & =\omega^{\prime}\left(t^{\prime}+T\left(x_{1}, x_{2}, \underline{x}_{3}^{0}\left(x_{1}, x_{2}, \mathbf{y}, t ; \mathbf{h}\right), \mathbf{h}, \mathbf{y}^{\prime}\right)\right) \\
& =-\omega^{\prime}(\underbrace{T\left(x_{1}, x_{2}, \underline{x}_{3}^{0}, \mathbf{h}, \mathbf{y}\right)}_{=t}-T\left(x_{1}, x_{2}, \underline{x}_{3}^{0}, \mathbf{h}, \mathbf{y}\right)
\end{aligned}
$$

$$
\begin{aligned}
& \left.-t^{\prime}+T\left(x_{1}, x_{2}, \underline{x}_{3}^{0}, \mathbf{h}, \mathbf{y}^{\prime}\right)\right) \\
= & -\omega^{\prime}\left(t-T\left(x_{1}, x_{2}, \underline{x}_{3}^{0}, \mathbf{h}, \mathbf{y}\right)\right. \\
& \left.-t^{\prime}+T\left(x_{1}, x_{2}, \underline{x}_{3}^{0}, \mathbf{h}, \mathbf{y}^{\prime}\right)\right) \\
= & \omega^{\prime}\left(t^{\prime}-t\right)+\omega^{\prime}\left(T\left(x_{1}, x_{2}, \underline{x}_{3}^{0}, \mathbf{h}, \mathbf{y}\right)\right. \\
& \left.-T\left(x_{1}, x_{2}, \underline{x}_{3}^{0}, \mathbf{h}, \mathbf{y}^{\prime}\right)\right) .
\end{aligned}
$$

We expand $T\left(x_{1}, x_{2}, x_{3}^{0}, \mathbf{h}, \mathbf{y}^{\prime}\right)$ in a Taylor series about the point $\mathbf{y}^{\prime}=\mathbf{y}$, which results in

$$
\begin{aligned}
& T\left(x_{1}, x_{2}, \underline{x}_{3}^{0}, \mathbf{h}, \mathbf{y}^{\prime}\right) \simeq T\left(x_{1}, x_{2}, \underline{x}_{3}^{0}, \mathbf{h}, \mathbf{y}\right) \\
& \quad+\left.\nabla_{y^{\prime}} T\left(x_{1}, x_{2}, \underline{x}_{3}^{0}, \mathbf{h}, \mathbf{y}^{\prime}\right)\right|_{\mathbf{y}^{\prime}=\mathbf{y}} \cdot\left(\mathbf{y}^{\prime}-\mathbf{y}\right)
\end{aligned}
$$

Thus, using the shorthand notation $\nabla_{y} T$ for $\nabla_{y^{\prime}} T\left(x_{1}, x_{2}, \underline{x}_{3}^{0}\right.$, $\left.\mathbf{h}, \mathbf{y}^{\prime}\right)\left.\right|_{\mathbf{y}^{\prime}=\mathbf{y}}$, we have

$$
\Phi \simeq \omega^{\prime}\left(t^{\prime}-t\right)+\omega^{\prime} \nabla_{y} T \cdot\left(\mathbf{y}-\mathbf{y}^{\prime}\right) .
$$

To obtain an explicit representation of a pseudodifferential operator, we change variables $\left(x_{1}, x_{2}\right) \rightarrow\left(\eta_{1}^{\prime}, \eta_{2}^{\prime}\right)$ in accordance with

$$
\boldsymbol{\eta}^{\prime}=\left.\omega^{\prime} \nabla_{y^{\prime}} T\left(x_{1}, x_{2}, \underline{x}_{3}^{0}\left(x_{1}, x_{2}, \mathbf{y}, t ; \mathbf{h}\right), \mathbf{h}, \mathbf{y}^{\prime}\right)\right|_{\mathbf{y}^{\prime}=\mathbf{y}}
$$

for given $(\mathbf{y}, t)$ and $\mathbf{h}$ still fixed. (Note that the $\underline{x}_{3}^{0}$ dependence contains implicitly a dependence on $x_{1}, x_{2}$.) With this definition, equation $\mathrm{C}-13$ gives equation $\mathrm{C}-3$. To compute the Jacobian associated with the transformation $\left(x_{1}, x_{2}\right) \rightarrow$ $\left(\eta_{1}^{\prime}, \eta_{2}^{\prime}\right)$, we compute

$$
\begin{aligned}
\mathbf{J} & =\frac{\partial\left(\eta_{1}^{\prime}, \eta_{2}^{\prime}\right)}{\partial\left(x_{1}, x_{2}\right)} \\
& =\frac{\omega^{\prime 2}}{c^{2}}\left|\begin{array}{ll}
\partial_{x_{1}} \eta_{1}^{\prime}+\partial_{x_{3}} \eta_{1}^{\prime} \frac{\partial \underline{x}_{3}^{0}}{\partial x_{1}} & \partial_{x_{2}} \eta_{1}^{\prime}+\partial_{x_{3}} \eta_{1}^{\prime} \frac{\partial \underline{x}_{3}^{0}}{\partial x_{2}} \\
\partial_{x_{1}} \eta_{2}^{\prime}+\partial_{x_{3}} \eta_{2}^{\prime} \frac{\partial \underline{x}_{3}^{0}}{\partial x_{1}} & \partial_{x_{2}} \eta_{2}^{\prime}+\partial_{x_{3}} \eta_{2}^{\prime} \frac{\partial \underline{x}_{3}^{0}}{\partial x_{2}}
\end{array}\right| .
\end{aligned}
$$

Introducing the vectors $\mathbf{p}=(\mathbf{x}-\mathbf{y}-\mathbf{h})$ and $\mathbf{q}=(\mathbf{x}-\mathbf{y}+\mathbf{h})$ and orienting the system of coordinates so that $\mathbf{h}=\left(h_{1}, 0,0\right)$, we have, upon substituting $T=(|\mathbf{q}|+|\mathbf{p}|) / c$,

$$
\begin{aligned}
& J=\frac{\omega^{\prime 2}}{c^{2}} \\
& \times\left|\begin{array}{cc}
\frac{-|\mathbf{q}|^{2}+q_{1}^{2}}{|\mathbf{q}|^{3}}+\frac{-|\mathbf{p}|^{2}+p_{1}^{2}}{|\mathbf{p}|^{3}}+\left(\frac{q_{1} q_{3}}{|\mathbf{q}|^{3}}+\frac{p_{1} p_{3}}{|\mathbf{p}|^{3}}\right) \frac{\partial \underline{\underline{x}}_{3}^{0}}{\partial x_{1}} & \frac{q_{1} q_{2}}{|\mathbf{q}|^{3}}+\frac{p_{1} p_{2}}{|\mathbf{p}|^{3}}+\left(\frac{q_{1} q_{3}}{|\mathbf{q}|^{3}}+\frac{p_{1} p_{3}}{|\mathbf{p}|^{3}}\right) \frac{\partial x_{3}^{0}}{\partial x_{2}} \\
\frac{q_{1} q_{2}}{|\mathbf{q}|^{3}}+\frac{p_{1} p_{2}}{|\mathbf{p}|^{3}}+\left(\frac{q_{1} q_{3}}{|\mathbf{q}|^{3}}+\frac{p_{1} p_{3}}{|\mathbf{p}|^{3}}\right) \frac{\partial \underline{x}_{3}^{0}}{\partial x_{1}} & \frac{-|\mathbf{q}|^{2}+q_{2}^{2}}{|\mathbf{q}|^{3}}+\frac{-|\mathbf{p}|^{2}+p_{2}^{2}}{|\mathbf{p}|^{3}}+\left(\frac{q_{2} q_{3}}{|\mathbf{q}|^{3}}+\frac{p_{2} p_{3}}{|\mathbf{p}|^{3}}\right) \frac{\partial \underline{x}_{3}^{0}}{\partial x_{2}}
\end{array}\right| .
\end{aligned}
$$

We solve equation C-4 for $\underline{x}_{3}^{0}$ :

$$
\begin{aligned}
\underline{x}_{3}^{0}= & \frac{1}{2 c t} \\
& \times \sqrt{-2 p_{1}^{2} c^{2} t^{2}-4 q_{2}^{2} c^{2} t^{2}+p_{1}^{4}+q_{1}^{4}-2 p_{1}^{2} q_{1}^{2}-2 q_{1}^{2} c^{2} t^{2}+c^{4} t^{4}},
\end{aligned}
$$

noting that $q_{2}=p_{2}$ and $q_{3}=p_{3}=\underline{x}_{3}^{0}$ and taking the positive square root as $x_{3}$ is constrained to be greater than zero. We 
then compute

$$
\begin{aligned}
\frac{\partial \underline{x}_{3}^{0}}{\partial x_{1}} & =\frac{k_{1}}{p_{3}}, \quad \text { where } \\
k_{1} & =\frac{-\left(p_{1}+q_{1}\right)\left(q_{2}^{2}+x_{3}^{2}+q_{1} q_{2}+|\mathbf{q}||\mathbf{p}|\right)}{c^{2} t^{2}} \\
\frac{\partial \underline{x}_{3}^{0}}{\partial x_{2}} & =\frac{-q_{2}}{p_{3}},
\end{aligned}
$$

where we again use the stationarity condition C-4 in the numerator of equation C-18. Substituting equations C-18 and C-19 into equation C-16 reduces the Jacobian matrix to

$$
\left|\begin{array}{cc}
-\frac{1}{|\mathbf{q}|}+\frac{q_{1}^{2}+q_{1} k_{1}}{|\mathbf{q}|^{3}}-\frac{1}{|\mathbf{p}|}+\frac{p_{1}^{2}+p_{1} k_{1}}{|\mathbf{p}|^{3}} & 0 \\
* & -\frac{1}{|\mathbf{q}|}-\frac{1}{|\mathbf{p}|}
\end{array}\right|
$$

where the asterisk represents a term that we do not need to compute since it is multiplied by 0 in the Jacobian. Thus, we have for the Jacobian

$$
\begin{aligned}
\mathbf{J}= & \frac{\omega^{\prime 2}}{c^{2}}\left(\frac{1}{|\mathbf{p}|^{2}}+\frac{1}{|\mathbf{q}|^{2}}-\frac{p_{1}^{2}+k_{1} p_{1}}{|\mathbf{p}|^{3}}\left(\frac{1}{|\mathbf{p}|}+\frac{1}{|\mathbf{q}|}\right)\right. \\
& \left.-\frac{q_{1}^{2}+k_{1} q_{1}}{|\mathbf{q}|^{3}}\left(\frac{1}{|\mathbf{p}|}+\frac{1}{|\mathbf{q}|}\right)+\frac{2}{|\mathbf{q}||\mathbf{p}|}\right) .
\end{aligned}
$$

After some algebra, it can be shown that

$$
-\frac{p_{1}^{2}+k_{1} p_{1}}{|\mathbf{p}|^{3}}\left(\frac{1}{|\mathbf{p}|}+\frac{1}{|\mathbf{q}|}\right)+\frac{1}{|\mathbf{q}||\mathbf{p}|}=\frac{\mathbf{p} \cdot \mathbf{q}}{|\mathbf{p}|^{3}|\mathbf{q}|},
$$

which gives

$$
\mathbf{J}=\frac{\omega^{\prime 2}}{c^{2}}\left(\frac{1}{|\mathbf{p}|^{2}}+\frac{1}{|\mathbf{q}|^{2}}+\frac{\mathbf{p} \cdot \mathbf{q}}{|\mathbf{p}|^{3}|\mathbf{q}|}+\frac{\mathbf{q} \cdot \mathbf{p}}{|\mathbf{q}|^{3}|\mathbf{p}|}\right) .
$$

Since we are going to map from $\left(x_{1}, x_{2}\right)$ to $\left(\eta_{1}^{\prime}, \eta_{2}^{\prime}\right)$, we require $J^{-1}$. Since $\mathbf{J} \neq 0$, we find that

$$
\mathbf{J}^{-1}=\frac{\partial\left(x_{1}, x_{2}\right)}{\partial\left(\eta_{1}^{\prime}, \eta_{2}^{\prime}\right)}=\frac{c^{2}\left(|\mathbf{q}|^{2}|\mathbf{p}|^{2}\right)}{\omega^{\prime 2}\left(|\mathbf{q}|^{2}+|\mathbf{p}|^{2}\right)(1+\cos \theta)},
$$

where $\theta$ is the angle between the source and receiver rays at the scattering point (i.e., $\left.\cos \theta=(\mathbf{q} \cdot \mathbf{p})|\mathbf{q}|^{-1}|\mathbf{p}|^{-1}\right)$.

\section{Treatment of amplitudes}

To compute the final amplitude function, we begin with the initial amplitude $\mathcal{A}$, the product of the imaging and modeling amplitudes:

$$
\mathcal{A}=\frac{1}{4 \pi^{2}} \omega^{\prime 2} \omega^{2} A(\mathbf{x}, \mathbf{s}, \mathbf{r}) A\left(\mathbf{x}, \mathbf{s}^{\prime}, \mathbf{r}^{\prime}\right),
$$

where $A(\mathbf{x}, \mathbf{s}, \mathbf{r})=1 /\left(8 \pi^{2} c^{3}|\mathbf{s}-\mathbf{x}||\mathbf{x}-\mathbf{r}|\right)$.

We now follow the same steps as performed on the phase, beginning by changing to the midpoint-offset coordinates and performing stationary phase in $\left(x_{3}, \omega\right)$, giving

$$
\begin{aligned}
\mathcal{A}^{\prime}= & \frac{1}{4 \pi^{2}} \omega^{\prime 4}\left(\frac{\partial_{x_{3}} T\left(x_{1}, x_{2}, \underline{x}_{3}^{0}, \mathbf{y}, \mathbf{h}\right)}{\partial_{x_{3}} T\left(x_{1}, x_{2}, \underline{x}_{3}^{0}, \mathbf{y}^{\prime}, \mathbf{h}\right)}\right)^{2} \\
& \times \frac{A\left(x_{1}, x_{2}, \underline{x}_{3}^{0}, \mathbf{y}, \mathbf{h}\right) A\left(x_{1}, x_{2}, \underline{x}_{3}^{0}, \mathbf{y}^{\prime}, \mathbf{h}\right)}{\sqrt{\left|\partial_{x_{3}} T\left(x_{1}, x_{2}, \underline{x}_{3}^{0}, \mathbf{y}, \mathbf{h}\right)\right|^{2}}} .
\end{aligned}
$$

Up to the principal part (for which symbols and amplitudes are equal), we can assume $\mathbf{y} \approx \mathbf{y}^{\prime}$ (Treves, 1980a, p. 37), giving

$$
\frac{1}{4 \pi^{2}} \frac{\omega^{\prime 4} A^{2}\left(x_{1}, x_{2}, \underline{x}_{3}^{0}, \mathbf{y}, \mathbf{h}\right)}{\left|\partial_{x_{3}} T\left(x_{1}, x_{2}, \underline{x}_{3}^{0}, \mathbf{y}, \mathbf{h}\right)\right|} \text {. }
$$

It is possible to write $\partial_{x 3} T$ in the notation defined above as

$$
\partial_{x_{3}} T=\frac{q_{3}}{c|\mathbf{q}|}+\frac{p_{3}}{c|\mathbf{p}|} \text {. }
$$

The product of the Jacobian of equation C-24 with $\partial_{x 3} T$ from equation C-28 gives equation B-25 of de Hoop et al. (1999).

Multiplying equation C-27 by the Jacobian $\partial\left(x_{1}, x_{2}\right) /$ $\partial\left(\eta_{1}^{\prime}, \eta_{2}^{\prime}\right)$ and combining with the phase yields the kernel of the final operator:

$$
\begin{gathered}
F F^{*}\left(\mathbf{y}, t, \mathbf{y}^{\prime}, t^{\prime}\right)=\frac{1}{4 \pi^{2}} \iiint \frac{\omega^{\prime 4} A^{2}\left(\underline{x}_{1}, \underline{x}_{2}, \underline{x}_{3}^{0}, \mathbf{y}, \mathbf{h}\right)}{\left|\partial_{x_{3}} T\left(\underline{x}_{1}, \underline{x}_{2}, \underline{x}_{3}^{0}, \mathbf{y}, \mathbf{h}\right)\right|} \\
\times \frac{\partial\left(x_{1}, x_{2}\right)}{\partial\left(\eta_{1}^{\prime}, \eta_{2}^{\prime}\right)} e^{i \omega^{\prime}\left(t^{\prime}-t\right)+i \eta^{\prime} \cdot\left(\mathbf{y}-\mathbf{y}^{\prime}\right)} \mathrm{d} \omega^{\prime} \mathrm{d} \eta_{1}^{\prime} \mathrm{d} \eta_{2}^{\prime}, \quad \text { (C-29) }
\end{gathered}
$$

where $\underline{x}_{1}$ and $\underline{x}_{2}$ are underlined because they are now functions of $\left(\eta_{1}^{\prime}, \eta_{2}^{\prime}\right)$. It can be shown that the amplitude of this operator is the reciprocal of that of Miller et al. (1987, their equation 27), upon changing variables in that equation from $\boldsymbol{\xi}$ to $\mathbf{y}$, using the Jacobian given in de Hoop et al. (1999). This amplitude construction can be used to correctly account for the amplitudes in offset continuation. For that case, one would fix both the output offset, $\mathbf{h}=(\mathbf{s}-\mathbf{r}) / 2$, and the input offset, $\mathbf{h}^{\prime}=\left(\mathbf{s}^{\prime}-\mathbf{r}^{\prime}\right) / 2$ and follow the same procedure as for the case $\mathbf{h}=\mathbf{h}^{\prime}$.

\section{REFERENCES}

Beylkin, G., 1985, Imaging of discontinuities in the inverse scattering problem by inversion of a causal generalized Radon transform: Journal of Mathematical Physics, 26, 99-108.

Biondi, B., S. Fomel, and N. Chemingui, 1998, Azimuth moveout for 3-D prestack imaging: Geophysics, 63, 574-588.

Black, J. L., K. L. Schleicher, and L. Zhang, 1993, True-amplitude imaging and dip moveout: Geophysics, 58, 47-66.

Bleistein, N., 1990, Born DMO revisited: 60th Annual International Meeting, SEG, Expanded Abstracts, 1366-1369.

Bleistein, N., and H. Jaramillo, 2000, A platform for data mapping in scalar models of data acquisition: Geophysical Prospecting, 48, 135-161.

Bleistein, N., J. K. Cohen, and H. Jaramillo, 1999, True-amplitude transformation to zero offset of data from curved reflectors: Geophysics, 64, 112-129.

Bleistein, N., J. K. Cohen, and J. W. Stockwell, Jr., 2000, Mathematics of multidimensional seismic imaging, migration and inversion: Springer-Verlag New York.

Brandsberg-Dahl, S., M. V. de Hoop, and B. Ursin, 2003a, Focusing in dip and AVA compensation on scattering-angle/azimuth gathers: Geophysics, 68, 232-254.

Brandsberg-Dahl, S., B. Ursin, and M. V. de Hoop, 2003b, Seismic velocity analysis in the scattering-angle/azimuth domain: Geophysical Prospecting, 51, 295-314.

Burridge, R., and G. Beylkin, 1988, On double integrals over spheres: Inverse Problems, 4, No. 1, 1-10. 
Burridge, R., M. V. de Hoop, D. Miller, and C. Spencer, 1998, Multiparameter inversion in anisotropic media: Geophysical Journal International, 134, 757-777.

Canning, A., and G. H. F. Gardner, 1996, Regularizing 3-D data sets with DMO: Geophysics, 61, 1103-1114.

Claerbout, J. F., 1985, Imaging the Earth's interior: Blackwell Scientific Publications.

de Hoop, M. V., and N. Bleistein, 1997, Micro-local, non-linear, resolution analysis of generalised Radon inversion in anisotropic media: Inverse Problems, 13, 669-690.

de Hoop, M. V., and S. Brandsberg-Dahl, 2000, Maslov asymptotic extension of generalized Radon transform inversion in anisotropic elastic media: A least squares approach: Inverse Problems, 16, 519-562

de Hoop, M. V., J. H. Le Rousseau, and B. Biondi, 2003a, Symplectic structure of wave-equation imaging: A path-integral approach based on the double-square-root equation: Geophysical Journal International, 153, 52-74.

de Hoop, M. V., A. E. Malcolm, and J. H. Le Rousseau, 2003b, Seismic wavefield 'continuation' in the single scattering approximation: A framework for dip and azimuth moveout: Canadian Applied Mathematics Quarterly, 10, 199-238.

de Hoop, M. V., C. Spencer, and R. Burridge, 1999, The resolving power of seismic amplitude data: An anisotropic inversion/ migration approach: Geophysics, 64, 852-873.

Deregowski, S. M., and F. Rocca, 1981, Geometrical optics and wave theory of constant offset sections in layered media: Geophysical Propspecting, 29, No. 3, 374-406.

Duistermaat, J. J., 1996, Fourier integral operators: vol. 130, Birkhäuser.

Fomel, S., 1995, Amplitude preserving offset continuation in theory, part 1: The offset continuation equation: Stanford Exploration Project Report 84, 179-196. 68, 718-732.

N. Bleistein, 2001, Amplitude preservation for offset continuation: Confirmation for Kirchhoff data: Journal of Seismic Exploration, 10, 121-130.

Fomel, S., and M. Prucha, 1999, Angle-gather time migration: Stanford Exploration Project Report 100, 141-150.

Foss, S.-K., M. V. de Hoop, and B. Ursin, 2003, Linearized 2.5-D parameter imaging-inversion in anisotropic elastic media: Center for Wave Phenomena preprint CWP 431P.

Fowler, P. J., 1998, A comparative overview of dip moveout methods: 68th Annual International Meeting, SEG, Expanded Abstracts, $1744-1747$.

Goldin, S., 1994, Superposition and continuation of transformations used in seismic migration: Russian Geology and Geophysics, 35, $131-145$
Goldin, S., and S. Fomel, 1995, Estimation of reflection coefficient in DMO: Russian Geology and Geophysics, 36, No. 4, 103-115.

Hale, D., 1991, Dip moveout processing: Course notes series, Society of Exploration Geophysicists.

Hörmander, L., 1985a, The analysis of linear partial differential operators, vol. 3: Springer-Verlag Berlin.

1985b, The analysis of linear partial differential operators, vol. 4: Springer-Verlag Berlin.

Hubral, P., J. Schleicher, and M. Tygel, 1996, A unified approach to 3-D seismic reflection imaging, part I: Basic concepts: Geophysics, 61, 742-758.

Liner, C. L., 1991, Born theory of wave-equation dip moveout: Geophysics, 56, 182-189.

Loewenthal, D. L. Lu, R. Roberson, and J. Sherwood, 1976, The wave equation applied to migration: Geophysical Prospecting, 24, No. 2, 380-399.

Miller, D., M. Oristaglio, and G. Beylkin, 1987, A new slant on seismic imaging: Migration and integral geometry: Geophysics, 52, 943 964.

Patch, S. K., 2002, Computation of unmeasured third-generation VCT views from measured views: IEEE Transactions on Medical Imaging, 21, No. 7, 801-813.

Santos, L. T., J. Schleicher, and M. Tygel, 1997, 2.5-D true amplitude offset continuation: Journal of Seismic Exploration, 6, No. 2/3, 103-116.

Sava, P., and S. Fomel, 2003, Angle-domain common-image gathers by wavefield continuation methods: Geophysics, $\mathbf{6 8}, 1065-1074$.

Sethian, J. A., 2002, Fast algorithms for optimal control, anisotropic front propagation and multiple arrivals: International Congress of Mathematicians, 3, 735-746.

Stolk, C. C., 2001, Microlocal analysis of the scattering angle transform: Preprint, Rice University, 7 September 2004 〈http://www.caam.rice.edu/ ${ }^{-}$cstolk/angle.ps .

Stolk, C. C., and M. V. de Hoop, 2002, Microlocal analysis of seismic inverse scattering in anisotropic elastic media: Communications on Pure and Applied Mathematics, 55, No. 3, 261-391.

Stolt, R. H., 2002, Seismic data mapping and reconstruction: Geophysics, 67, 890-908.

ten Kroode, A., D. Smit, and A. Verdel, 1998, A microlocal analysis of migration: Wave Motion, 28, 149-172.

Treves, F., 1980a, Introduction to pseudodifferential and Fourier integral operators, vol. 1: Plenum Press.

1980b, Introduction to pseudodifferential and Fourier integral operators, vol. 2: Plenum Press.

Tygel, M., J. Schleicher, P. Hubral, and L. T. Santos, 1998, 2.5-D trueamplitude Kirchhoff migration to zero offset in laterally inhomogeneous media: Geophysics, 63, 557-573.

White, B. S., B. Nair, and A. Bayliss, 1988, Random rays and seismic amplitude anomalies: Geophysics, 53, 903-907. 